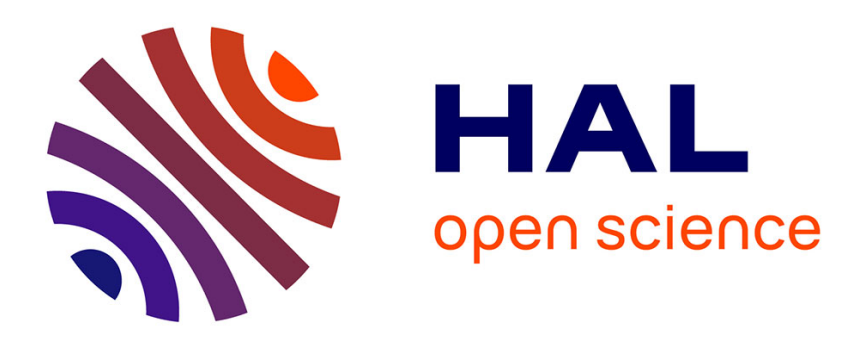

\title{
Effective Erdös-Wintner theorems for digital expansions
}

Michael Drmota, Johann Verwee

\section{To cite this version:}

Michael Drmota, Johann Verwee. Effective Erdös-Wintner theorems for digital expansions. 2021. hal-03189795

\section{HAL Id: hal-03189795 \\ https://hal.science/hal-03189795}

Preprint submitted on 5 Apr 2021

HAL is a multi-disciplinary open access archive for the deposit and dissemination of scientific research documents, whether they are published or not. The documents may come from teaching and research institutions in France or abroad, or from public or private research centers.
L'archive ouverte pluridisciplinaire HAL, est destinée au dépôt et à la diffusion de documents scientifiques de niveau recherche, publiés ou non, émanant des établissements d'enseignement et de recherche français ou étrangers, des laboratoires publics ou privés. 


\title{
EFFECTIVE ERDÖS-WINTNER THEOREMS FOR DIGITAL EXPANSIONS
}

\author{
MICHAEL DRMOTA*, AND JOHANN VERWEE*
}

\begin{abstract}
In 1972 Delange [8] observed in analogy of the classical Erdős-Wintner theorem that $q$-additive functions $f(n)$ has a distribution function if and only if the two series $\sum f\left(d q^{j}\right)$, $\sum f\left(d q^{j}\right)^{2}$ converge. The purpose of this paper is to provide quantitative versions of this theorem as well as generalizations to other kinds of digital expansions. In addition to the $q$-ary and Cantor case we focus on the Zeckendorf expansion that is based on the Fibonacci sequence, where we provide a sufficient and necessary condition for the existence of a distribution function, namely that the two series $\sum f\left(F_{j}\right), \sum f\left(F_{j}\right)^{2}$ converge (previously only a sufficient condition was known $[1])$.
\end{abstract}

\section{INTRODUCTION}

The classical Erdős-Wintner theorem $[13,14,17,16]$ states that a real valued additive function $f$, that is defined by the property $f(m n)=f(m)+f(n)$ for coprime positive integers $m, n$, has a distribution function

$$
F(y)=\lim _{N \rightarrow \infty} \frac{1}{N} \#\{n<N: f(n) \leqslant y\}
$$

if and only if the following three series converge:

$$
\sum_{|f(p)|>1} \frac{1}{p}, \quad \sum_{|f(p)| \leqslant 1} \frac{f(p)}{p}, \quad \sum_{|f(p)| \leqslant 1} \frac{f(p)^{2}}{p} .
$$

This is a proper analogue of Kolmogorov's three series theorem in probability theory. Recently Tenenbaum and the second author have studied effective versions of this theorem [32].

The purpose of the present paper is to consider the analogue problem in the context of digital expansions, where we restrict ourselves to the (common) $q$-ary digital expansion, to Cantor digital expansions, and to the Zeckendorf expansion. Both, the prime decomposition as well as digital expansions are expansions of integers. Whereas the prime decomposition encodes the multiplicative structure, digital expansions encode - in some way - the additive structure of the integers.

Note that a function is additive if and only if

$$
f\left(p_{1}^{e_{1}} \cdots p_{r}^{e_{r}}\right)=f\left(p_{1}^{e_{1}}\right)+\cdots+f\left(p_{r}^{e_{r}}\right),
$$

where $p_{1}, \ldots, p_{r}$ are different primes and $e_{1}, \ldots, e_{r}$ positive integers.

In a similar way one defines so-called $q$-additive functions by

$$
f\left(d_{1} q^{e_{1}}+\cdots+d_{r} q^{e_{r}}\right)=f\left(d_{1} q^{e_{1}}\right)+\cdots+f\left(d_{r} q^{e_{r}}\right),
$$

where $q \geqslant 2$ is a given integer, $e_{1}<e_{2}<\cdots<e_{r}$ are different positive integers and $d_{1}, \ldots, d_{r}$ are integers digits satisfying $1 \leqslant d_{j} \leqslant q-1(1 \leqslant j \leqslant r)$.

For example, the $q$-ary sum-of-digits function $s_{q}(n)$, defined by $s_{q}\left(d q^{a}\right)=d$, is $q$-additive as well as the $q$-ary Van-der-Corput sequence $v_{q}(n)$, defined by $v_{q}\left(d q^{a}\right)=d q^{-a-1}$.

The analysis of $q$-additive functions and their distribution (as well as their multiplicative counterpart) have attained a lot of attention during the last few decades, see for example $[3,6,7,9,10,11,18,21,22,23,24,25,26,30]$.

As already indicated, in the present paper we want to focus on analogues of the Erdős-Wintner theorems for digital expansions and their quantitative versions. For the $q$-adic case there is already a

* TU Wien, Institute of Discrete Mathematics and Geometry, Wiedner Hauptstrasse 8-10, A-1040 Vienna, Austria. michael.drmota@tuwien.ac.at.johann.verwee@tuwien.ac.at. Research supported by the Austrian Science Foundation FWF, project F55-02.

Keywords: Erdős-Wintner theorem, digital expansions. 
proper analogue by Delange [8] saying that a real-valued $q$-additive function $f(n)$ has a distribution function $F(y)$ if and only if the two series

$$
\sum_{j \geqslant 0} \sum_{d=1}^{q-1} f\left(d q^{j}\right) \text { and } \sum_{j \geqslant 0} \sum_{d=1}^{q-1} f\left(d q^{j}\right)^{2}
$$

converge. By applying a Berry-Esseen inequality we present a quantified version of this theorem and give examples, the most interesting one is related to Cantor-Lebesgue measures (Section 2).

The results for $q$-ary expansions can be easily extended to Cantor digital expansions with bounded quotients (Section 3).

Finally we discuss the Zeckendorf digital expansions, where the base sequence are the Fibonacci numbers (Section 4). This is actually the most challenging case. First of all we give a full characterization for the existence of a distribution function (so far only a sufficient condition was known [1]). This requires a delicate analysis of Fibonacci-like recurrences with non-constant coefficients. A quantitative version can be then established under more general hypotheses.

\section{2. $q$-ARY Digital EXPANSIONS}

We start with a self-contained proof of the Theorem 1 by Delange [8] since we will use similar principles later. We then give a quantitative version (Section 2.2) and after an example we discuss Cantor-Lebesgue measures (Section 2.3).

\subsection{The $q$-ary Erdös-Wintner theorem.}

Theorem 1 (Delange [8]). Let $f(n)$ be a real-valued $q$-additive function. Then $f(n)$ has a distribution function $F(y)$, that is

$$
\lim _{N \rightarrow \infty} \frac{1}{N} \#\{n<N \mid f(n) \leqslant y\}=F(y),
$$

if and only if the two series

$$
\sum_{j \geqslant 0} \sum_{d=1}^{q-1} f\left(d q^{j}\right) \quad \text { and } \quad \sum_{j \geqslant 0} \sum_{d=1}^{q-1} f\left(d q^{j}\right)^{2}
$$

converge. In this case the characteristic function $\varphi(t)$ of the limiting distribution is given by

$$
\varphi(t)=\int_{-\infty}^{\infty} e^{i t y} \mathrm{~d} F(y)=\prod_{j \geqslant 0}\left(\frac{1}{q} \sum_{d=0}^{q-1} e^{i t f\left(d q^{j}\right)}\right) .
$$

Before (re-)proving Theorem 1 we mention that a theorem of Jessen and Wintner asserts that such a distribution measure given by $F(y)$ is pure (that is, it is either absolutely continuous having a density or purely singular continuous, where $F$ is continuous and has zero derivative almost everywhere, or consists only of point masses). Note that the last alternative can only occur if and only if there exists an integer $J>0$ such that $f\left(d q^{j}\right)=0$ for $j \geqslant J$ and all $d \in\{1, \ldots q-1\}$ : in this case the distribution consists only of finitely many point masses, see [12, Lemma 1.22].

Proof. By Lévy's theorem it is sufficient to show that the characteristic functions converge for every fixed $t \in \mathbb{R}:$

$$
\lim _{N \rightarrow \infty} \frac{1}{N} \sum_{n<N} e^{i t f(n)}=\varphi(t),
$$

where the limit function $\varphi(t)$ is continuous at $t=0$. Since

$$
\varphi_{q^{L}}(t):=\frac{1}{q^{L}} \sum_{n<q^{L}} e^{i t f(n)}=\prod_{j=0}^{L-1}\left(\frac{1}{q} \sum_{d=0}^{q-1} e^{i t f\left(d q^{j}\right)}\right)
$$

it is clear that the limit has to be

$$
\varphi(t)=\prod_{j \geqslant 0}\left(\frac{1}{q} \sum_{d=0}^{q-1} e^{i t f\left(d q^{j}\right)}\right) .
$$

(Note that we are using the convention $f(0)=0$.) 
Set

$$
m_{j, q}:=\frac{1}{q} \sum_{d=1}^{q-1} f\left(d q^{j}\right), \quad m_{2 ; j, q}^{2}:=\frac{1}{q} \sum_{d=1}^{q-1} f\left(d q^{j}\right)^{2} .
$$

Then by using the relation $e^{i u}=1+i u+O\left(u^{2}\right)$ for real $u$ we have

$$
\begin{aligned}
\log \left(\frac{1}{q} \sum_{d=0}^{q-1} e^{i t f\left(d q^{j}\right)}\right) & =\log \left(1+i t m_{j, q}+O\left(t^{2} m_{2 ; j, q}^{2}\right)\right) \\
& =i t m_{j, q}+O\left(t^{2}\left(m_{j, q}^{2}+m_{2 ; j, q}^{2}\right)\right) \\
& =i t m_{j, q}+O\left(t^{2} m_{2 ; j, q}^{2}\right)
\end{aligned}
$$

provided that $j$ is sufficiently large (depending on $t$ ). Note thay by Cauchy-Schwarz's inequality

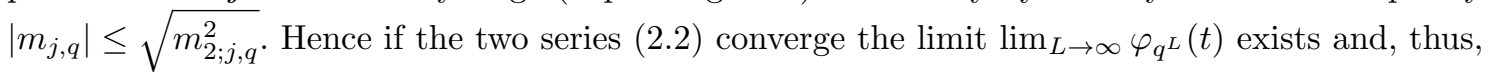
equals $\varphi(t)$. It is an easy exercise (by using the $q$-ary expansion of $N$, see also Lemma 3 ) that the convergence $\varphi_{q^{L}}(t) \rightarrow \varphi(t)$ implies the convergence (2.4), too. Finally it is not difficult to check that $\varphi(t)$ is continuous at $t=0$ (later we will even show that $\varphi(t)=1+O(t)$ as $t \rightarrow 0$ ).

Conversely, suppose that we have $\lim _{L \rightarrow \infty} \varphi_{q^{L}}(t)=\varphi(t)$, where $\varphi(t)$ is continuous at $t=0$. Clearly we have, too, $\lim _{L \rightarrow \infty}\left|\varphi_{q^{L}}(t)\right|=|\varphi(t)|$, and we can choose $t_{0}>0$ such that $|\varphi(t)| \geqslant \frac{1}{2}$ for all $t \in\left[0, t_{0}\right]$. We now use the fact that $1-\cos (x)=2 \sin ^{2}(x / 2) \geqslant 8\|x /(2 \pi)\|^{2}$ (where $\|u\|$ denotes the distance from $u$ to the set of integers) and obtain

$$
\begin{aligned}
\left|\sum_{d=0}^{q-1} e^{i t f\left(d q^{j}\right)}\right|^{2} & =\sum_{d_{1}, d_{2}=0}^{q-1} \cos \left(t\left(f\left(d_{1} q^{j}\right)-f\left(d_{2} q^{j}\right)\right)\right) \\
& \leqslant q^{2}-2 \sum_{d=0}^{q-1}\left(1-\cos \left(t f\left(d q^{j}\right)\right)\right) \\
& \leqslant q^{2}-16 \sum_{d=0}^{q-1}\left\|\frac{t f\left(d q^{j}\right)}{2 \pi}\right\|^{2}
\end{aligned}
$$

and

$$
\left|\frac{1}{q} \sum_{d=0}^{q-1} e^{i t f\left(d q^{j}\right)}\right| \leqslant \exp \left(-\frac{8}{q^{2}} \sum_{d=0}^{q-1}\left\|\frac{t f\left(d q^{j}\right)}{2 \pi}\right\|^{2}\right) .
$$

Now, if $0<t \leqslant t_{0}$ we obtain

$$
\frac{1}{2} \leqslant|\varphi(t)|=\prod_{j \geqslant 0}\left|\frac{1}{q} \sum_{d=0}^{q-1} e^{i t f\left(d q^{j}\right)}\right| \leqslant \exp \left(-\frac{8}{q^{2}} \sum_{j \geqslant 0} \sum_{d=0}^{q-1}\left\|\frac{t f\left(d q^{j}\right)}{2 \pi}\right\|^{2}\right)
$$

and, thus,

$$
\sum_{j \geqslant 0} \sum_{d=0}^{q-1}\left\|\frac{t f\left(d q^{j}\right)}{2 \pi}\right\|^{2} \leqslant c_{1}
$$

for some constant $c_{1}>0$. In particular it follows that the sequence $\left\|t f\left(d q^{j}\right) /(2 \pi)\right\|^{2}(1 \leqslant d \leqslant q-1$, $j \geqslant 0)$ converges to 0 for all $t \in\left(0, t_{0}\right]$. Actually this also implies that $f\left(d q^{j}\right)$ converges to $0 .{ }^{1}$ In particular the sequence $f\left(d q^{j}\right)$ is bounded. Thus, we can choose $t \in\left(0, t_{0}\right]$ such that $\left|t f\left(d q^{j}\right)\right| \leqslant \pi$ for all $d$ and $j$. By (2.7) this implies that the sum

$$
\sum_{j \geqslant 0} \sum_{d=0}^{q-1}\left(\frac{t f\left(d q^{j}\right)}{2 \pi}\right)^{2}
$$

is bounded and consequently convergent for $t \in\left(0, t_{0}\right]$. Since $t>0$ we also get convergence of the $\operatorname{sum} \sum_{j \geqslant 0} \sum_{d=0}^{q-1} f\left(d q^{j}\right)^{2}$.

\footnotetext{
${ }^{1}$ For the reader's convenience we append a proof of this property in the Appendix.
} 
Now the final step is easy. By using the fact that $\lim _{L \rightarrow \infty} \varphi_{q^{L}}(t)=\varphi(t)$ and by taking logarihms (as above) it follows that the sum

$$
\sum_{j \geqslant 0} m_{j, q}=\frac{1}{q} \sum_{j \geqslant 0} \sum_{d=1}^{q-1} f\left(d q^{j}\right)
$$

converges, too.

2.2. An Effective Version of the $q$-ary Erdös-Wintner Theorem. Clearly, every $q$-additive function is determined by the values $f\left(d q^{j}\right)$, where $d \leqslant q-1$ and $j \geqslant 0$. There is one very simple case that we briefly discuss first. Suppose that only finitely many values $f\left(d q^{j}\right)$ are non-zero, that is, there exists $J>0$ such that $f\left(d q^{j}\right)=0$ for $j \leqslant J$ and all $d \leqslant q-1$. Then $f(n)$ is periodic with period $q^{J}$ and by $(2.3)$ the limiting distribution function $F$ equals $F_{q^{J}}$. Furthermore, if we write $N=q^{J} Q+r$ for some $0 \leqslant r<q^{J}$ it follows that

$$
\#\{n<N: f(n) \leqslant y\}=Q \#\left\{n<q^{J}: f(n) \leqslant y\right\}+\#\{n<r: f(n) \leqslant y\}
$$

which implies

$$
F_{N}(y)=\frac{1}{N} \#\{n<N: f(n) \leqslant y\}=\frac{Q q^{J}}{N} F(y)+O\left(\frac{q^{J}}{N}\right)=F(y)+O\left(\frac{q^{J}}{N}\right) .
$$

In different terms

$$
\left\|F_{N}-F\right\|_{\infty} \ll \frac{1}{N}
$$

which is the optimal convergence rate (the implicit constant depends of $f$, of course).

From now on we will assume that $f\left(d q^{j}\right) \neq 0$ for infinitely many instances.

Theorem 2. Let $f(n)$ be a real-valued q-additive function such that the two series (2.2) converge and that $f\left(d q^{j}\right) \neq 0$ for infinitely many instances. Set $L=\left\lfloor\log _{q} N\right\rfloor$. Then we have for all real numbers $T \geqslant 1$ such that $h=\left\lceil\log _{q}(T \log T)\right\rceil \leqslant L$

$$
\left\|F_{N}-F\right\|_{\infty} \ll Q_{F}\left(\frac{1}{T}\right)+T\left|\sum_{j>L} \sum_{d=0}^{q-1} f\left(d q^{j}\right)\right|+T \sqrt{h} \sqrt{\sum_{j \geqslant L-h} \sum_{d=0}^{q-1} f\left(d q^{j}\right)^{2}},
$$

where $F_{N}$ denotes the distribution function of $(f(n): n<N)$, $F$ the limiting distribution function, and

$$
Q_{F}(h)=\sup _{z \in \mathbb{R}}(F(z+h)-F(z)) \quad(h>0) .
$$

The implicit constant depends on $q$.

Note that the upper bound (2.8) refers explicitly to the two series (2.2) and goes to 0 with $N \rightarrow \infty$ if these two series converge and if $Q_{F}(h) \rightarrow 0$ as $h \rightarrow 0$ (and with $T$ going slowly enough to $+\infty)$. Actually, we can improve the bound (2.8) by

$$
\begin{aligned}
\left\|F_{N}-F\right\|_{\infty} & \ll Q_{F}\left(\frac{1}{T}\right)+T \sum_{j=L-h+1}^{L} \sum_{d=0}^{q-1}\left|f\left(d q^{j}\right)\right| \\
& +T\left|\sum_{j>L} \sum_{d=0}^{q-1} f\left(d q^{j}\right)\right|+T^{2} \sum_{j>L} \sum_{d=0}^{q-1} f\left(d q^{j}\right)^{2}
\end{aligned}
$$

that is slightly better but also more involved. (Clearly by applying Cauchy-Schwarz's inequality and the bound $|u| \ll u^{2}$ for bounded $u$ (2.10) implies (2.8).)

It seems there is no simple and tight upper bound for $Q_{F}(h)$ in terms of $f$. Clearly, if $F$ is absolutely continuous and $F^{\prime}$ is bounded, then $Q_{F}(h) \ll h$ (which is by the way optimal since we always have $Q_{F}(h) \gg h$ for $\left.0 \leq h<1\right)$. If $\varphi \in L^{p}(\mathbb{R})$, then we can use the following upper bound (see [31, lemme III.2.9])

$$
Q_{F}(h) \ll h \int_{-1 / h}^{1 / h}|\varphi(t)| \mathrm{d} t .
$$

and Hölder's inequality to obtain $Q_{F}(h) \ll h^{1 / p}$. 
In general we can only use proper estimates for $|\varphi(t)|$ that are deduced from the product representation (2.3) and (2.11). In particular this leads to (2.16) (see Lemma 1). By applying this bound and by doing also some modifications in order to simplify it for applications we formulate the following variant. This variant applies in particular if the series

$$
\sum_{j \geqslant 0} \frac{1}{q} \sum_{d=0}^{q-1}\left|f\left(d q^{j}\right)\right|
$$

converges and does not necessarily give an upper bound that tends to zero if the two series (2.2) converge.

Theorem 3. Let $f(n)$ be a real-valued $q$-additive function such that the series (2.12) converge and that $f\left(d q^{j}\right) \neq 0$ for infinitely many instances. Set

$$
S(t)=\left\{(d, j) \in\{1, \ldots, q-1\} \times \mathbb{N}:\left|f\left(d q^{j}\right)\right| \leqslant \pi /|t|\right\}(t>0) .
$$

Then with $c_{1}=2 /\left(\pi^{2} q^{2}\right)$ and the abbreviations $L=\left\lfloor\log _{q} N\right\rfloor$ and $h=\left\lceil\log _{q}(T \log T)\right\rceil$, we have for all real numbers $T \geqslant 1$ such that $h \leqslant L$

$$
\begin{aligned}
\left\|F_{N}-F\right\|_{\infty} & \ll \frac{1}{T} \int_{0}^{T} \exp \left(-c_{1} t^{2} \sum_{(d, j) \in S(t)} f\left(d q^{j}\right)^{2}\right) \mathrm{d} t+T \sum_{j=L-h+1}^{L} \sum_{d=0}^{q-1}\left|f\left(d q^{j}\right)\right| \\
& +\int_{1 / T}^{T} \min \left\{\frac{1}{1+t}, \sum_{j>L} \sum_{d=0}^{q-1}\left|f\left(d q^{j}\right)\right|\right\} \exp \left(-c_{1} t^{2} \sum_{(d, j) \in S(t), j \leqslant L} f\left(d q^{j}\right)^{2}\right) \mathrm{d} t
\end{aligned}
$$

where $F_{N}$ denotes the distribution function of $(f(n): n<N)$ and $F$ the limiting distribution function (the implicit constant depending on $q$ ).

We note that the sums

$$
t^{2} \sum_{(d, j) \in S(t)} f\left(d q^{j}\right)^{2}
$$

could be replaced by the better bound

$$
\frac{8}{q^{2}} \sum_{j \geqslant 0} \sum_{d=0}^{q-1}\left\|\frac{t f\left(d q^{j}\right)}{2 \pi}\right\|^{2}
$$

see the proof of Lemma 1 below (clearly if $(d, j) \in S(t)$ then $\left\|t f\left(d q^{j}\right) /(2 \pi)\right\|^{2}=t^{2} f\left(d q^{j}\right)^{2} /\left(4 \pi^{2}\right)$, and the other terms are just neglected). However, the sum (2.14) is easier to handle than the sum (2.15). Nevertheless, there are cases, where we need the better bound (2.15), see Section 2.3.

Our analysis is based on the Berry-Esseen inequality [12, Lemma 1.47] that we state first:

Proposition 1. Let $F$ and $G$ be two distributions functions of probability distributions with characteristic functions $\varphi$ and $\psi$, respectively. Then we have for all $T>0$

$$
\|F-G\|_{\infty} \ll Q_{F}\left(\frac{1}{T}\right)+\int_{-T}^{T}\left|\frac{\varphi(t)-\psi(t)}{t}\right| \mathrm{d} t .
$$

In order to apply the Berry-Esseen inequality we need some information on the characteristic functions $\varphi(t)$ and $\varphi_{N}(t)$. The proofs of Theorems 2 and 3 will be done in parallel. We first give an upper bound of $\varphi(t)$ in terms of $f$.

Lemma 1. Let $\varphi(t)$ be the characteristic function given by (2.3). Then (with the notation of Theorem 2) we have

$$
|\varphi(t)| \leqslant \exp \left(-\frac{2}{\pi^{2} q^{2}} t^{2} \sum_{(d, j) \in S(t)} f\left(d q^{j}\right)^{2}\right) .
$$


Proof. From (2.6) we directly obtain

$$
\begin{aligned}
|\varphi(t)| & \leqslant \exp \left(-\frac{8}{q^{2}} \sum_{j \geqslant 0} \sum_{d=0}\left\|\frac{t f\left(d q^{j}\right)}{2 \pi}\right\|^{2}\right) \\
& \leqslant \exp \left(-\frac{2}{\pi^{2} q^{2}} t^{2} \sum_{(d, j) \in S(t)} f\left(d q^{j}\right)^{2}\right) .
\end{aligned}
$$

Next we consider the difference $\varphi(t)-\varphi_{N}(t)$ for $|t| \leqslant 1 / T$. For this purpose we recall the definitions of $m_{j, q}$ and $m_{2 ; j, q}$ from (2.5). It is easy to obtain the following two representations:

$$
\frac{1}{q^{L}} \sum_{n<q^{L}} f(n)=\sum_{\ell<L} m_{\ell, q}, \quad \frac{1}{q^{L}} \sum_{n<q^{L}} f(n)^{2}=\sum_{\ell<L}\left(m_{2 ; \ell, q}^{2}-m_{\ell, q}^{2}\right)+\left(\sum_{\ell<L} m_{\ell, q}\right)^{2} .
$$

From this we easily obtain the following property.

Lemma 2. Suppose that $f(n)$ is a real-valued q-additive function such that the two series $(2.2)$ converge. Then we have

$$
\frac{1}{N} \sum_{n<N}|f(n)|=O(1)
$$

as $N \rightarrow \infty$; the implicit constant depends on $f$.

Proof. Suppose that $L=\left\lceil\log _{q} N\right\rceil$. Then $N \leqslant q^{L}<q N$ and we have

$$
\begin{aligned}
\frac{1}{N} \sum_{n<N} f(n)^{2} & \leqslant \frac{q}{q^{L}} \sum_{n<q^{L}} f(n)^{2} \\
& =q \sum_{\ell<L}\left(m_{2 ; \ell, q}^{2}-m_{\ell, q}^{2}\right)+q\left(\sum_{\ell<L} m_{\ell, q}\right)^{2} \\
& =O(1) .
\end{aligned}
$$

Recall that $m_{j, q}^{2} \leqslant m_{2 ; j, q}^{2}$. Consequently by Cauchy-Schwarz's inequality

$$
\frac{1}{N} \sum_{n<N}|f(n)| \leqslant\left(\frac{1}{N} \sum_{n<N} f(n)^{2}\right)^{1 / 2}=O(1)
$$

which completes the proof of the lemma.

By definition and the elementary inequality $\left|e^{i t}-1\right| \leqslant|t|$ we have

$$
\begin{aligned}
\left|1-\varphi_{N}(t)\right| & =\left|\frac{1}{N} \sum_{n<N}\left(1-e^{i t f(n)}\right)\right| \\
& \leqslant|t| \frac{1}{N} \sum_{n<N}|f(n)| \\
& =O(|t|) .
\end{aligned}
$$

By taking the limit $N \rightarrow \infty$ we also obtain $|1-\varphi(t)|=O(t)$. This also implies that $\varphi_{N}(t)-\varphi(t)=$ $O(t)$ and consequently the ratio $\frac{1}{t}\left(\varphi_{N}(t)-\varphi(t)\right)$ stays bounded. In particular, the following integral can be trivially bounded:

$$
\int_{-1 / T}^{1 / T}\left|\frac{\varphi_{N}(t)-\varphi(t)}{t}\right| \mathrm{d} t \ll \frac{1}{T} .
$$

Note that $Q_{F}(1 / T) \gg 1 / T$. Thus we can neglect the term $1 / T$.

Finally we have to deal with $\varphi(t)-\varphi_{N}(t)$ for $1 / T<|t| \leqslant T$. Since we have no direct access to $\varphi_{N}$ we have to approximate it by $\varphi_{q}{ }^{L}$, where $L=\left\lfloor\log _{q} N\right\rfloor$ and where we can apply the following lemma by Delange [8, Proposition 3] (properly adjusted). 
Lemma 3. Suppose that $f(n)$ is real-valued and q-additive. Then we have for all integers $h \geqslant 1$ and $N \geqslant q^{h}$

$$
\left|\varphi_{N}(t)-\varphi_{q^{L+1}}(t)\right| \leqslant \frac{2}{q^{h-1}}+2 \sqrt{2} \sum_{j=L-h+1}^{L} \max _{1 \leq d \leq q-1}\left(1-\cos \left(t f\left(d q^{j}\right)\right)^{1 / 2}\right.
$$

where $L=\left\lfloor\log _{q} N\right\rfloor$.

We note that [8] states the slightly worse bound

$$
\left|\varphi_{N}(t)-\varphi_{q^{L+1}}(t)\right| \leqslant \frac{2}{q^{h-1}}+2 \sqrt{2 h}\left(\sum_{j=L-h+1}^{L} \max _{1 \leqslant d \leqslant q-1}\left(1-\cos \left(t f\left(d q^{j}\right)\right)\right)\right)^{1 / 2}
$$

The final step in [8] is to apply the Cauchy-Schwarz inequality so that (2.17) implies (2.18).

Since $1-\cos (x)=2 \sin ^{2}(x / 2) \leqslant x^{2} / 2$ we, thus, obtain

$$
\int_{1 / T<|t| \leqslant T} \frac{\left|\varphi_{N}(t)-\varphi_{q^{L+1}}(t)\right|}{|t|} \mathrm{d} t \ll \frac{\log T}{q^{h}}+T \sum_{j=L-h+1}^{L} \sum_{d=0}^{q-1}\left|f\left(d q^{j}\right)\right| .
$$

The choice $h=\left\lceil\log _{q}(T \log T)\right\rceil$ ensures that $(\log T) / q^{h} \leqslant 1 / T$.

What remains is to consider the corresponding integral over the difference $\left|\varphi_{q^{L+1}}(t)-\varphi(t)\right|$. Obviously we have

$$
\varphi_{q^{L+1}}(t)-\varphi(t)=\varphi_{q^{L+1}}(t)\left(1-\frac{\varphi(t)}{\varphi_{q^{L+1}}(t)}\right)
$$

where

$$
\frac{\varphi(t)}{\varphi_{q^{L+1}}(t)}=\prod_{j>L}\left(\frac{1}{q} \sum_{d=0}^{q-1} e^{i t f\left(d q^{j}\right)}\right)
$$

We can either use the trivial estimate $\left|\varphi_{q^{L+1}}(t)\right| \leq 1$ or proceed as in Lemma 1 to get

$$
\left|\varphi_{q^{L+1}}(t)\right| \leqslant \exp \left(-\frac{2}{\pi^{2} q^{2}} t^{2} \sum_{(d, j) \in S(t), j \leqslant L} f\left(d q^{j}\right)^{2}\right) .
$$

Furthermore, we trivially have $\left|1-\varphi(t) / \varphi_{q^{L+1}}(t)\right| \leqslant 2$ and (by the properties $\varphi(t)=1+O(t)$ and $\varphi_{q^{L+1}}(t)=1+O(t)$ as $\left.t \rightarrow 0\right)\left|1-\varphi(t) / \varphi_{q^{L+1}}(t)\right|=O(t)$. This implies

$$
\frac{\left|1-\varphi(t) / \varphi_{q^{L+1}}(t)\right|}{|t|} \ll \frac{1}{1+|t|} .
$$

By using the properties $e^{i u}=1+i u+O\left(u^{2}\right),\left|e^{i u}-1\right| \leq|u|$ and $1+|u| \leq e^{|u|}$ (for real $u$ ) we have

$$
\prod_{j>L}\left(\frac{1}{q} \sum_{d=0}^{q-1} e^{i t f\left(d q^{j}\right)}\right)=\exp \left(i t \sum_{j>L} \frac{1}{q} \sum_{d=0}^{q-1} f\left(d q^{j}\right)+O\left(t^{2} \sum_{j>L} \frac{1}{q} \sum_{d=0}^{q-1} f\left(d q^{j}\right)^{2}\right)\right)
$$

and, thus,

$$
\begin{aligned}
\left|\prod_{j>L}\left(\frac{1}{q} \sum_{d=0}^{q-1} e^{i t f\left(d q^{j}\right)}\right)-1\right| & \leqslant\left|\exp \left(c t^{2} \sum_{j>L} \frac{1}{q} \sum_{d=0}^{q-1} f\left(d q^{j}\right)^{2}\right)-1\right| \\
& +\left|t \sum_{j>L} \frac{1}{q} \sum_{d=0}^{q-1} f\left(d q^{j}\right)\right| .
\end{aligned}
$$


Since the left hand side is upper bounded by 2 this also implies

$$
\begin{aligned}
\left|\prod_{j>L}\left(\frac{1}{q} \sum_{d=0}^{q-1} e^{i t f\left(d q^{j}\right)}\right)-1\right| & \leqslant c^{\prime} t^{2} \sum_{j>L} \frac{1}{q} \sum_{d=0}^{q-1} f\left(d q^{j}\right)^{2} \\
& +\left|t \sum_{j>L} \frac{1}{q} \sum_{d=0}^{q-1} f\left(d q^{j}\right)\right|
\end{aligned}
$$

for some constant $c^{\prime}>0$. This directly provides the upper bound

$$
\int_{1 / T<|t| \leqslant T} \frac{\left|\varphi_{q^{L+1}}(t)-\varphi(t)\right|}{|t|} \mathrm{d} t \ll T\left|\sum_{j>L} \frac{1}{q} \sum_{d=0}^{q-1} f\left(d q^{j}\right)\right|+T^{2} \sum_{j>L} \frac{1}{q} \sum_{d=0}^{q-1} f\left(d q^{j}\right)^{2}
$$

and completes the proof of (2.10) and consequently that of Theorem 2 .

Finally, by using the inequality

$$
\left|\prod_{k=1}^{K} a_{k}-1\right| \leqslant \sum_{k=1}^{K}\left|a_{k}-1\right|
$$

for complex numbers $a_{k}$ with $\left|a_{k}\right| \leqslant 1$, we have

$$
\begin{aligned}
\left|\prod_{j>L}\left(\frac{1}{q} \sum_{d=0}^{q-1} e^{i t f\left(d q^{j}\right)}\right)-1\right| & \leqslant \sum_{j>L}\left|\frac{1}{q} \sum_{d=0}^{q-1} e^{i t f\left(d q^{j}\right)}-1\right| \\
& \leqslant \frac{|t|}{q} \sum_{j>L} \sum_{d=0}^{q-1}\left|f\left(d q^{j}\right)\right|
\end{aligned}
$$

provided that the last sum converges. Clearly this estimate completes the proof of Theorem 3 .

Example. Suppose that $q=2$ and that

$$
c_{1} j^{-\alpha} \leqslant f\left(2^{j}\right) \leqslant c_{2} j^{-\alpha}
$$

for $j \geqslant 1$ and some $\alpha$ that satisfies $1<\alpha<2$, where $c_{1}, c_{2}$ are positive constants. For this kind of asymptotic behavior we obtain

$$
\left\|F-F_{N}\right\|_{\infty} \ll(\log N)^{1-\alpha}
$$

as we will see in a moment.

First we have

$$
\sum_{j \in S(t)} f\left(2^{j}\right)^{2} \geqslant \sum_{j>c_{3}|t|^{1 / \alpha}} j^{-2 \alpha} \geqslant c_{4}|t|^{-2+1 / \alpha}
$$

for proper positive constants $c_{3}, c_{4}$. Consequently

$$
\int_{-\infty}^{\infty} \exp \left(-c t^{2} \sum_{j \in S(t)} f\left(2^{j}\right)^{2}\right) \mathrm{d} t=O(1) .
$$

Thus, the first term in Theorem 3 is bounded by $O(1 / T)$.

Next we set $T=L^{\alpha-1}$, where $L=\left\lfloor\log _{2} N\right\rfloor$. and $h=\left\lceil\log _{2}(T \log T)\right\rceil=(\alpha-1) \log _{2} L+$ $O(\log \log L)$. In particular this implies that

$$
\frac{\log T}{2^{h}} \ll \frac{\log L}{L^{\alpha}} \ll L^{1-\alpha}
$$

and (since $\alpha<2$ )

$$
T \sum_{j=L-h+1}^{L}\left|f\left(2^{j}\right)\right| \ll L^{\alpha-1}(\log L) L^{-\alpha} \ll \frac{\log L}{L} \ll L^{1-\alpha}
$$

Finally we have

$$
\sum_{j>L}\left|f\left(2^{j}\right)\right| \ll L^{1-\alpha}
$$


and for $|t| \geqslant 1 / T=L^{1-\alpha}$ we have

$$
\sum_{j \in S(t), j \leqslant L} f\left(2^{j}\right)^{2} \geqslant \sum_{c_{3}|t|^{1 / \alpha}<j \leqslant L} j^{-2 \alpha} \geqslant c_{4}\left(|t|^{-2+1 / \alpha}-L^{-2 \alpha+1}\right) \geqslant c_{5}|t|^{-2+1 / \alpha} .
$$

which implies that also the last term in Theorem 3 is bounded by $L^{1-\alpha}$.

If $\alpha \geqslant 2$ we can do a similar analysis and obtain $\left\|F-F_{N}\right\|_{\infty} \ll \sqrt{\log \log N}(\log N)^{-\alpha / 2}$.

2.3. Cantor-Lebesgue Measures. We now discuss the binary case $q=2$ and the 2-additive function $f(n)$ that is given by

$$
f\left(2^{j}\right)=\beta^{j} \quad(j \geqslant 0)
$$

for some $\beta \in(0,1)$. In all cases the limiting distribution $F=F_{\beta}$ is continuous, however, it can be quite wild in general. Let $\mu_{\beta}$ denote the corresponding limiting measure. It is easy to see that $\mu_{\beta}$ is linked to the distribution of $\sum_{n=0}^{\infty} \pm \beta^{n}$ (where the signs are chosen independently with probability $1 / 2)$, denoted by $\nu_{\beta}$, by the relation

$$
\mu_{\beta}(B)=\nu_{\beta}\left(2 B-\frac{1}{1-\beta}\right)
$$

for any real Borel set $B \in \mathcal{B}(\mathbb{R})$. We just have to compare the corresponding characteristic functions.

The following quote is from [27]:

Kershner and Wintner (1935) observed that $\nu_{\beta}$ is singular for $\beta \in(0,1 / 2)$ since it is supported on a Cantor set of zero Lebesgue measure (in fact, $\nu_{\beta}$ is the standard Cantor-Lebesgue measure on this Cantor set). Wintner (1935) noted that $\nu_{\beta}$ is uniform on $[-2,2]$ for $\beta=1 / 2$ and for $\beta=(2-1 / k)^{-1}$ with $k \geqslant 2$ it is absolutely continuous, with a density in $C^{k-2}(\mathbb{R})$. For $\beta \in(1 / 2,1)$ the support of $\nu_{\beta}$ is the interval $\left[-(1-\beta)^{-1},(1-\beta)^{-1}\right]$, so one might surmise that $\nu_{\beta}$ is absolutely continuous for all such $\beta$. However, in [15] Erdős (1939) showed that is singular when $\beta$ is the reciprocal of a Pisot number (recall that a Pisot number is an algebraic integer all of whose conjugates are less than one in modulus). This gives a closed countable set of $\beta \in(1 / 2,1)$ with $\nu_{\beta}$ singular.

In the case $f\left(2^{j}\right)=\beta^{j}$ we cannot apply Theorem 3 directly since the (easy) upper bound for $|\varphi(t)|$ is not sufficient to obtain any bound that tends to zero. Nevertheless, a more careful analysis provides the following bound.

Theorem 4. Let $f(n)$ be the 2-additive function defined by $f\left(2^{j}\right)=\beta^{j}, j \geqslant 0$, where $\beta \in(0,1)$. Then we have

$$
\left\|F-F_{N}\right\| \ll_{\beta} N^{-c(\beta)}(\log N)^{\log (1 / \beta) / \log 2}
$$

for some exponent $c(\beta)>0^{2}$ (and the implicit constant depends on $\beta$, too). Moreover, if $F$ is absolutely continuous, then we have

$$
\left\|F-F_{N}\right\| \ll_{\beta} N^{-\log (1 / \beta) /(\log 2+\log (2 / \beta))}(\log N)^{\log (1 / \beta) / \log 2} .
$$

We note that these bounds are certainly not optimal, since $c=c(\beta)$ that we get from the following proof is usually a very small number. On the other hand, for $\beta=\frac{1}{2}$ the resulting sequence is the Van-der-Corput sequence that has discrepancy of (optimal) order $(\log N) / N$ which is much better than what we obtain in Theorem 4. In general we expect that optimal bounds are of the form $N^{-\tilde{c}(\beta)+o(1)}$ for some $\tilde{c}(\beta)>0$.

In the present case the characteristic function is given by

$$
\varphi(t)=\prod_{j \geqslant 0} \frac{1+e^{i t \beta^{j}}}{2}
$$

and consequently

$$
|\varphi(t)|=\prod_{j \geqslant 0}\left(\frac{1+\cos \left(t \beta^{j}\right)}{2}\right)^{1 / 2} \leqslant \exp \left(-\frac{1}{2} \sum_{j \geqslant 0}\left\|t \beta^{j} / \pi\right\|^{2}\right) .
$$

\footnotetext{
${ }^{2}$ Gérald Tenenbaum mentioned to us that a different approach to the concentration function leads to the estimation $\left\|F-F_{N}\right\| \ll N^{-\bar{c}(\beta)}(\log N)^{\log (1 / \beta) / \log 2}$ with a relatively simple explicit number $\bar{c}(\beta)$. We will discuss this in the Appendix B.
} 
It is essential to include the terms $\left\|t \beta^{j} / \pi\right\|$ for which $\left|t \beta^{j}\right|>\pi / 2$ although they behave quite erratic. However, in the average they contribute essentially and this we will use.

Set $B=1 / \beta>1$ and suppose that $T>1$. Let $J_{0} \geqslant 0$ be the integer defined by $B^{J_{0}} \leqslant T<B^{J_{0}+1}$ Then we have

$$
\begin{aligned}
\int_{1}^{T}|\varphi(t)| \mathrm{d} t & \leqslant \sum_{J=0}^{J_{0}} \int_{B^{J}}^{B^{J+1}} \exp \left(-\frac{1}{2} \sum_{j \geqslant 0}\left\|t \beta^{j} / \pi\right\|^{2}\right) \mathrm{d} t \\
& =\sum_{J=0}^{J_{0}} B^{J} \int_{1}^{B} \exp \left(-\frac{1}{2} \sum_{0 \leqslant j \leqslant J}\left\|\tau B^{J-j} / \pi\right\|^{2}\right) \mathrm{d} \tau
\end{aligned}
$$

The most important ingredient for the proof of Theorem 4 is the following property.

Lemma 4. Suppose that $B>1$ and set $($ for $J \geqslant 0)$

$$
S_{J}(\tau)=\frac{1}{2} \sum_{0 \leqslant j \leqslant J}\left\|\tau B^{j} / \pi\right\|^{2}
$$

Then there exists $\eta>0$ (depending on $\beta$ ) such that:

$$
\int_{1}^{B} \exp \left(-S_{J}(\tau)\right) \mathrm{d} \tau \ll \exp (-\eta J)
$$

Proof. The idea of the proof is that the average value of $\frac{1}{2}\|x\|^{2}$ is $1 / 24$. Thus, we can expect that for most $\tau$ we have $S_{J}(\tau) \geqslant \eta J$ for some $\eta>0$. This would lead to (2.19). However, it is not that easy to make this heuristic argument rigorous. For this purpose we adopt methods from [4].

We first assume $B \geqslant 2$ (the proof is very similar for $1<B<2$, even slightly easier). We set

$$
f(x)=\frac{1}{24}-\frac{\|x\|^{2}}{2}=\sum_{k=1}^{\infty} c_{k} \cos (2 \pi k x) \quad(x \in \mathbb{R})
$$

where

$$
c_{k}=\frac{(-1)^{k+1}}{2 \pi^{2} k^{2}}
$$

Furthermore, let $H \geqslant 1$ and $\gamma \geqslant 2$ be two integers such that

$$
B^{4 H}-2 H^{\gamma} B^{3 H}-2 \pi B^{2 H}+2 \pi \geqslant 0 .
$$

We also put for $x \in \mathbb{R}$ and $m \in \mathbb{N}$

$$
g(x)=\sum_{k=1}^{H^{\gamma}} c_{k} \cos (2 \pi k x) \quad \text { and } \quad U_{m}(x)=\sum_{j=H m+1}^{H(m+1)} g\left((B-1) B^{j} x / \pi\right) .
$$

For any $\lambda>0, \psi \in\{f, g\}$, and $M \in \mathbb{N}$ we define

$$
\overline{S_{M, \psi}}(t)=\sum_{j=0}^{M} \psi\left(\frac{(B-1) B^{j}}{\pi} t+\frac{B^{j}}{\pi}\right)
$$

and

$$
\chi_{\psi}(\lambda, M)=(B-1) \int_{0}^{1} \exp \left(\lambda \overline{S_{M, \psi}}(t)\right) \mathrm{d} t
$$

We have the relation

$$
\int_{1}^{B} \exp \left(-S_{J}(\tau)\right) \mathrm{d} \tau=\exp \left(-\frac{J+1}{24}\right) \chi_{f}(1, J) .
$$

In order to estimate $\chi_{f}(1, J)$ we introduce a parameter $\lambda>0$ and consider $\chi_{f}(\lambda, J)$. Clearly we have the following inequality for any $\kappa>0$,

$$
\chi_{f}(1, J) \leqslant(B-1) \exp \left(\frac{J+1}{24}\right) \Lambda\left(\left\{t \in[0,1]:\left|\overline{S_{J, f}}(t)\right| \geqslant \kappa\right\}\right)+(B-1) \mathrm{e}^{\kappa},
$$


where $\Lambda$ denotes the Lebesgue measure. By Cernov's bound we have all $\lambda>0$

$$
\Lambda\left(\left\{t \in[0,1]:\left|\overline{S_{J, f}}(t)\right| \geqslant \kappa\right\}\right) \leqslant \frac{1}{B-1} \mathrm{e}^{-\lambda \kappa} \chi_{f}(\lambda, J),
$$

which implies

$$
\int_{1}^{B} \exp \left(-S_{J}(\tau)\right) \mathrm{d} \tau \leqslant \mathrm{e}^{-\lambda \kappa} \chi_{f}(\lambda, J)+(B-1) \exp \left(\kappa-\frac{J+1}{24}\right) .
$$

Since $|f(x)-g(x)| \leqslant \pi^{-2} H^{-\gamma}$ we can estimate $\chi_{f}(\lambda, J)$ with the help of $\chi_{g}(\lambda, J)$ :

$$
\chi_{f}(\lambda, J) \leqslant \exp \left(\frac{\lambda J}{\pi^{2} H^{\gamma}}\right) \chi_{g}(\lambda, J) .
$$

It remains to bound $\chi_{g}$. For simplicity we assume that $J=p H$, where $p$ is an even integer. Thus, from the representation

$$
\overline{S_{J, g}}(t)=\sum_{m=0}^{p / 2} U_{2 m}\left(t+\frac{1}{B-1}\right)+\sum_{m=1}^{p / 2} U_{2 m-1}\left(t+\frac{1}{B-1}\right)
$$

and an application of the Cauchy-Schwarz inequality we obtain

$$
\chi_{g}(\lambda, H p) \leqslant\left(\int_{0}^{1} \exp \left(\lambda \sum_{m=0}^{p / 2} U_{2 m}(t+\xi)\right) \mathrm{d} t\right)^{1 / 2}\left(\int_{0}^{1} \exp \left(\lambda \sum_{m=1}^{p / 2} U_{2 m-1}(t+\xi)\right) \mathrm{d} t\right)^{1 / 2} .
$$

In order to estimate these two integrals, we properly adapt the ideas and results of [4], together with an observation due to Hartman [20] and a lemma of an article by Takahashi [29]. One first key step is to use the inequality $e^{x} \leqslant\left(1+x+x^{2}\right) e^{|x|^{3}}, x \in \mathbb{R}$, to get

$$
\begin{aligned}
\int_{0}^{1} \exp \left(\lambda \sum_{m=0}^{p / 2} U_{2 m}(t+\xi)\right) \mathrm{d} t \ll \int_{-\infty}^{\infty}\left(\frac{\sin t}{t}\right)^{2} \exp \left(\lambda \sum_{m=0}^{p / 2} U_{2 m}(t+\xi)\right) \mathrm{d} t \\
\ll \exp \left(C_{1} \lambda^{3} p H^{3}\right) \int_{-\infty}^{\infty}\left(\frac{\sin t}{t}\right)^{2} \prod_{m=0}^{p / 2}\left(1+\frac{\lambda U_{2 m}(t+\xi)}{2}+\frac{\lambda^{2} U_{2 m}(t+\xi)^{2}}{4}\right) \mathrm{d} t .
\end{aligned}
$$

Then by using the Fourier expansion of $U_{2 m}(t)$ and the fact that

$$
\int_{-\infty}^{\infty}\left(\frac{\sin t}{t}\right)^{2} \cos (u(t+\alpha)) \mathrm{d} t=0
$$

for all real $\alpha$ and real $u \geqslant 2$ if follows - we skip the technical details - that for some $C_{2}>0$

$$
\int_{-\infty}^{\infty}\left(\frac{\sin t}{t}\right)^{2} \prod_{m=0}^{p / 2}\left(1+\frac{\lambda U_{2 m}(t+\xi)}{2}+\frac{\lambda^{2} U_{2 m}(t+\xi)^{2}}{4}\right) \mathrm{d} t \ll\left(1+C_{2} \lambda^{2} H\right)^{p / 2}
$$

and consequently

$$
\int_{0}^{1} \exp \left(\lambda \sum_{m=0}^{p / 2} U_{2 m}(t+\xi)\right) \mathrm{d} t \ll \exp \left(C_{1} \lambda^{3} J H^{2}+C_{2} \lambda^{2} J\right) .
$$

This leads then to

$$
\chi_{g}(\lambda, J) \ll \exp \left(C_{1} \lambda^{3} J H^{2}+C_{2} \lambda^{2} J\right)
$$

and by $(2.21)$ and $(2.20)$ to

$$
\int_{1}^{B} \exp \left(-S_{J}(\tau)\right) \mathrm{d} \tau \ll \exp \left(\frac{-J}{24}+\frac{\kappa}{2}\right)+\exp \left(\frac{\lambda J}{\pi^{2} H^{\gamma}}+C_{2} \lambda^{2} J+C_{1} \lambda^{3} J H^{2}-\lambda \kappa\right) .
$$

By choosing $\kappa=J / 25$ and $H$ and $\gamma$ sufficiently large as well as $\lambda>0$ sufficiently small such that

$$
\frac{1}{\pi^{2} H^{\gamma}}+C_{2} \lambda+C_{1} \lambda^{2} H^{2} \leqslant \frac{1}{50}
$$

we obtain the proposed result with $\eta=\min \{1 / 600, \lambda / 50\}$. 
A direct application of Lemma 4 gives (where we assume without loss of generality that $B>e^{\eta}$ )

$$
\begin{aligned}
Q_{F}(1 / T) & \ll \frac{1}{T} \int_{-T}^{T}|\varphi(t)| \mathrm{d} t \\
& \ll \frac{1}{T}\left(1+\sum_{J=0}^{J_{0}}(B \exp (-\eta))^{J}\right) \\
& \ll \frac{1}{T}(B \exp (-\eta))^{J_{0}} \\
& \ll T^{-\eta / \log B}
\end{aligned}
$$

Furthermore we have

and

$$
\sum_{j=L-h+1}^{L}\left|f\left(2^{j}\right)\right| \ll \beta^{L-h}
$$

so that Theorem 3 gives

$$
\sum_{j>L}\left|f\left(2^{j}\right)\right| \ll \beta^{L}
$$

$$
\left\|F-F_{N}\right\| \ll T^{-\eta / \log (1 / \beta)}+T \beta^{L-h} \leqslant T^{-\eta / \log (1 / \beta)}+T N^{-\frac{\log (1 / \beta)}{\log 2}}(T \log T)^{\frac{\log (1 / \beta)}{\log 2}} .
$$

Hence by choosing $T=N^{c_{0}(\beta)}$ where $c_{0}=c_{0}(\beta)=\log (1 / \beta)^{2} /(\eta \log 2+\log (1 / \beta) \log (2 / \beta))$ we obtain $\left\|F-F_{N}\right\| \ll N^{-c(\beta)}(\log N)^{\log (1 / \beta) / \log 2}$ with $c(\beta)=\eta c_{0} / \log (1 / \beta)$ as proposed.

Moreover, if $F$ is absolutely continuous, then $Q_{F}(1 / T) \ll 1 / T$ and we obtain the second upper bound.

\section{Cantor Digital Expansions}

The purpose of this part is to state an effective Erdős-Wintner theorem for Cantor numeration system. In fact, the results and the proofs are very similar to the previous case.

We start by recalling the construction of a Cantor numeration system: we choose a sequence $\left(a_{n}\right)_{n \geqslant 0}$ of integers such that $a_{n} \geqslant 2$ for all $n$. The so-called Cantor numeration system $Q$ is thus the sequence $\left(q_{n}\right)_{n \geqslant 0}$ defined by $q_{0}=1$ and $q_{n+1}=a_{n} q_{n}$ for all $n \geqslant 0$, hence $q_{n}=a_{n-1} \cdots a_{1} a_{0}$. Then every nonnegative integer $n$ has a unique expansion

$$
n=\sum_{j \geqslant 0} \delta_{j}(n) q_{j}, \quad 0 \leqslant \delta_{j}(n) \leqslant a_{j}-1 .
$$

The length of $N$ is thus defined by $L=L(N):=\max \left\{j \geqslant 0: q_{j} \leqslant N<q_{j+1}\right\}$.

Clearly this type of system is a generalization of the $q$-adic case by taking $a_{n}=q \geqslant 2$ for all $n$. Furthermore we define $Q$-additive function by

$$
f\left(d_{1} q_{e_{1}}+\cdots+d_{r} q_{e_{r}}\right)=f\left(d_{1} q_{e_{1}}\right)+\cdots+f\left(d_{r} q_{e_{r}}\right),
$$

where $e_{1}<e_{2}<\cdots<e_{r}$ are different positive integers and $d_{1}, \ldots, d_{r}$ are integers digits satisfying $1 \leqslant d_{j} \leqslant q_{j}-1(1 \leqslant j \leqslant r)$.

For example, the Van-der-Corput sequence $v_{Q}(n)$ related to the Cantor numberation system $Q$ is defined by $v_{Q}\left(d q_{a}\right)=d q_{a+1}^{-1}$.

A Cantor numeration system is said to be constant-like if the sequence $\left(a_{n}\right)$ is bounded.

3.1. Erdős-Wintner theorem for constant-like Cantor numeration system. In [5] Coquet proved an Erdös-Wintner theorem for $Q$-additive functions when $\left(a_{n}\right)$ is bounded.

Theorem 5. Let $f(n)$ be a real valued $Q$-additive function with respect to a constant-like Cantor numeration system. Then $f(n)$ has a distribution function $F(y)$, that is

$$
\lim _{N \rightarrow \infty} \frac{1}{N} \#\{n<N \mid f(n) \leqslant y\}=F(y),
$$

if and only if the two series

$$
\sum_{j \geqslant 0} \frac{1}{a_{j}} \sum_{d=1}^{a_{j}-1} f\left(d q_{j}\right) \quad \text { and } \sum_{j \geqslant 0} \frac{1}{a_{j}} \sum_{d=1}^{a_{j}-1} f\left(d q_{j}\right)^{2}
$$


converge. In this case the characteristic function $\varphi(t)$ of the limiting distribution is given by

$$
\varphi(t)=\int_{-\infty}^{\infty} e^{i t y} \mathrm{~d} F(y)=\prod_{j \geqslant 0}\left(\frac{1}{a_{j}} \sum_{d=0}^{a_{j}-1} e^{i t f\left(d q_{j}\right)}\right) .
$$

It can be proved in the same way as in the $q$-adic (by using Lévy's theorem). We note that Barat and Grabner [2] wrote an alternative proof from ergodic point of view where they could avoid "the original Fourier analysis techniques".

We also note that distribution measure given by $F(y)$ is pure and that it consists only of finitely many point masses if and only if there exists a $J$ such that $f\left(d q_{j}\right)=0$ for $j>J$ and all $d \in\left\{1, \ldots, a_{j}-1\right\}$ (see [2, Proposition 3]).

3.2. An Effective Erdős-Wintner theorem for constant-like Cantor numeration system. As in the $q$-adic case, if only finitely many values $f\left(d q_{j}\right)$ are non-zero, then $f(n)$ is periodic with period $q_{J}$ and by (3.3) the limiting distribution function $F$ equals $F_{q_{J}}$ and we obtain

$$
\left\|F_{N}-F\right\|_{\infty} \ll \frac{1}{N}
$$

which is (again) the optimal convergence rate.

From now on we will assume that $f\left(d q_{j}\right) \neq 0$ for infinitely many instances. By doing the same reasoning as for the proof we obtain the following result.

Theorem 6. Let $f(n)$ be a real-valued $Q$-additive function (with respect to a constant-like Cantor numeration system) such that the two series (3.2) converge and $f\left(d q_{j}\right) \neq 0$ for infinitely many instances. Let $L=L(N)$ denote the length of $N$ and $h=\left\lceil\log _{a}(T \log T)\right\rceil$ (where a is the minimum of $\left.\left(a_{n}\right)\right)$. Then we have for all real numbers $T \geqslant 1$ such that $h \leqslant L(N)$

$$
\left\|F_{N}-F\right\|_{\infty} \ll Q_{F}\left(\frac{1}{T}\right)+T\left|\sum_{j>L} \frac{1}{a_{j}} \sum_{d=0}^{a_{j}-1} f\left(d q_{j}\right)\right|+T \sqrt{h} \sqrt{\sum_{j \geqslant L-h} \frac{1}{a_{j}} \sum_{d=0}^{a_{j}-1} f\left(d q_{j}\right)^{2}},
$$

where $F_{N}$ denotes the distribution function of $(f(n): n<N)$ and $F$ the limiting distribution function. All implicit constants depend on the minimum and maximum of the sequence $\left(a_{n}\right)_{n}$.

We note that the same remarks as given for Theorems 2 and 3 apply here, too. In particular we can state an analogue to Theorem 3:

$$
\begin{aligned}
\left\|F_{N}-F\right\|_{\infty} & \ll \frac{1}{T} \int_{0}^{T} \exp \left(-c_{2} t^{2} \sum_{(d, j) \in S_{C}(t)} \frac{f\left(d q_{j}\right)^{2}}{a_{j}^{2}}\right) \mathrm{d} t+T \sum_{j=L-h+1}^{L} \frac{1}{a_{j}} \sum_{d=0}^{a_{j}-1}\left|f\left(d q_{j}\right)\right| \\
& +\int_{1 / T}^{T} \min \left\{\frac{1}{1+t}, \sum_{j>L} \frac{1}{a_{j}} \sum_{d=0}^{a_{j}-1}\left|f\left(d q_{j}\right)\right|\right\} \exp \left(-c_{2} t^{2} \sum_{(d, j) \in S_{C}(t), j \leqslant L} \frac{f\left(d q_{j}\right)^{2}}{a_{j}^{2}}\right) \mathrm{d} t
\end{aligned}
$$

where

$$
S_{C}(t)=\left\{(d, j) \in\left\{1, \ldots, a_{j}-1\right\} \times \mathbb{N}:\left|f\left(d q_{j}\right)\right| \leqslant \pi /|t|\right\}
$$

and $c_{2}=2 / \pi^{2}$. We do not give the details, we only note that we used the fact that $\left(a_{n}\right)$ is bounded to obtain a result similar to Lemma 2 (which explains the dependence on the sequence $\left(a_{n}\right)_{n}$ in Theorem 6). And we also state the following result that is analogous to Lemma 3.

Lemma 5. Suppose that $f(n)$ is real-valued and $Q$-additive. Then we have for all integers $h \geqslant 1$ and $N \geqslant q_{\lceil h\rceil}$

$$
\left|\varphi_{N}(t)-\varphi_{q_{L+1}}(t)\right| \leqslant \frac{2}{a_{L-h+1} \cdots a_{L-1}}+2 \sqrt{2} \sum_{j=L-h+1}^{L} \max _{1 \leq d \leq a_{j}-1}\left(1-\cos \left(t f\left(d q_{j}\right)\right)^{1 / 2}\right.
$$

where $L=L(N)$ is the length of $N$.

Example. The $Q$-additive function

$$
v_{Q}(n)=\sum_{k=0}^{\infty} \frac{\delta_{k}(n)}{q_{k+1}}
$$


defines the van der Corput sequence for a Cantor numeration system $\left(a_{n}\right)_{n}$. Let assume that the sequence $\left(a_{n}\right)_{n}$ is bounded. Then the two series (3.2) certainly converge. It is also possible to compute the characteritic function of the limiting distribution

$$
\begin{aligned}
\Phi(t) & =\prod_{j=0}^{\infty} \frac{1}{a_{j}}\left(1+\sum_{d=1}^{a_{j}-1} \exp \left(\frac{i t d}{q_{j+1}}\right)\right) \\
& =\prod_{j=0}^{\infty}\left(\frac{\exp \left(\frac{i t}{2 q_{j}}-\frac{i t}{2 q_{j+1}}\right)}{a_{j}} \frac{\sin \left(t / 2 q_{j}\right)}{\sin \left(t / 2 q_{j+1}\right)}\right) \\
& =e^{\frac{i t}{2}} \prod_{j=0}^{\infty} \frac{2 q_{j} \sin \left(t / 2 q_{j}\right)}{2 q_{j+1} \sin \left(t / 2 q_{j+1}\right)} \\
& =e^{\frac{i t}{2}} \frac{\sin (t / 2)}{t / 2}
\end{aligned}
$$

that is precisely the characteristic function of the uniform distribution on $[0,1]$. In particular, $F$ is absolutely continuous with density $F^{\prime}(z)=1$ (for $0<z<1$ ). In particular $Q_{F}(h)=\min (h, 1)$.

This corresponds to the well-known fact that the Van-der-Corput sequence is uniformly distribution modulo 1 . The distance $\left\|F-F_{N}\right\|_{\infty}$ is then precisely the discrepancy, for which it is known that

$$
\left\|F-F_{N}\right\|_{\infty} \ll \frac{\log N}{N} .
$$

We can find more general and specific results in [19], for example. By applying the methods used in the proof of Theorem 6 , we only obtain

$$
\left\|F-F_{N}\right\|_{\infty} \ll \frac{\log N}{N^{1 / 3}}
$$

where we have used that fact that $Q_{F}(h)=\min (h, 1)$ and where we choose $T=N^{1 / 3}$.

3.3. A Partial Erdős-Wintner Theorem for General Cantor Numeration Systems. It is a natural question what can be said for unbounded sequences $\left(a_{n}\right)$. In [5], Conquet stated a sufficient condition for the existence of a limit distribution.

Proposition 2 (Coquet [5]). Let $f$ be a real-valued $Q$-additive function. We set, for all $j \geqslant 0$ and $d \in\left\{1, \ldots, a_{j}-1\right\}$

$$
f^{*}\left(d q_{j}\right)= \begin{cases}f\left(d q_{j}\right), & \text { if }\left|f\left(d q_{j}\right)\right| \leqslant 1 \\ 1, & \text { if }\left|f\left(d q_{j}\right)\right|>1\end{cases}
$$

and

$$
\beta_{j}:=\max _{1 \leqslant k \leqslant a_{j}-1}\left(\frac{1}{k+1} \sum_{d=0}^{k} f^{*}\left(d q_{j}\right)\right)^{2} .
$$

If $\beta_{j} \rightarrow 0$, and the series

$$
\sum_{j \geqslant 0} \frac{1}{a_{j}} \sum_{d=1}^{a_{j}-1} f^{*}\left(d q_{j}\right) \quad \text { and } \sum_{j \geqslant 0} \frac{1}{a_{j}} \sum_{d=1}^{a_{j}-1} f^{*}\left(d q_{j}\right)^{2}
$$

converge, then $f$ has a limit distribution and its characteristic function is

$$
\varphi(t)=\prod_{j=0}^{\infty}\left(\frac{1}{a_{j}} \sum_{d=0}^{a_{j}-1} \exp \left(i t f\left(d q_{j}\right)\right)\right) .
$$

We already mentioned that Barat and Grabner [2] proved Theorem 5 with the help of ergodic means. Actually they relate it to a convergence property of a series $\sum f_{n}(x)$ that is defined on the $Q$-adic integers $x \in \mathbb{Z}_{Q}=\lim _{\leftarrow} \mathbb{Z} / q_{n} \mathbb{Z}$. However, in the non-constant-like case they observe (with the help of a counter-example) that this relation is no longer an equivalence. It remains an open problem to formulate an Erdős-Wintner theorem for general Cantor systems. 


\section{ZeCKendorf Digital Expansions}

For $k \geqslant 0$ let $F_{k}$ be the $k$-th Fibonacci number, that is, $F_{0}=0, F_{1}=1$ and $F_{k}=F_{k-1}+F_{k-2}$ for $k \geqslant 2$. By Zeckendorf's theorem every positive integer $n$ admits a unique representation

$$
n=\sum_{i=2}^{L} \delta_{i}(n) F_{i}
$$

where $\delta_{i}(n) \in\{0,1\}$ and $\delta_{i}(n)=1$ implies $\delta_{i+1}(n)=0 ; L$ denotes the length of this expansion. This is by the way the optimal representation of $n$ as the sum of Fibonacci numbers in the sense that the number of Fibonacci numbers is minimal. We also recall that the Fibonacci numbers are explicitly given by

$$
F_{k}=\frac{1}{\sqrt{5}}\left(\gamma^{k}+(-1)^{k-1} \gamma^{-k}\right),
$$

where $\gamma=(1+\sqrt{5}) / 2$ is the golden number that satisfies the equation $\gamma^{2}=1+\gamma$.

A function $f$ on the non-negative integers is called Z-additive if

$$
f(n)=\sum_{i=2}^{L} f\left(\delta_{i}(n) F_{i}\right)
$$

that is, $f$ is uniquely determined by $f(0)=0$ and the values $f\left(F_{i}\right), i \geqslant 2$.

4.1. The Zeckendorf Erdős-Wintner theorem. Our first result is a proper version of the Erdős-Wintner theorem for Z-additive functions. We note that a partial result was given by [1].

Theorem 7. Let $f(n)$ be a real-valued $Z$-additive function. Then $f(n)$ has a distribution function $F(y)$ if and only if the two series

$$
\sum_{j \geqslant 2} f\left(F_{j}\right) \quad \text { and } \sum_{j \geqslant 2} f\left(F_{j}\right)^{2}
$$

converge. In this case the characteristic function $\varphi(t)$ of the limiting distribution is given by

$$
\varphi(t)=\frac{\sqrt{5}}{\gamma} \prod_{j \geqslant 2} \frac{r_{j}(t)}{\gamma},
$$

where $r_{2}(t)=1$ and

$$
r_{j+1}(t)=1+\frac{e^{i t f\left(F_{j}\right)}}{r_{j}(t)} \quad(j \geqslant 2)
$$

provided that $r_{j}(t) \neq 0$ for all $j \geqslant 2$.

We note that the limiting distribution is purely atomic if and only if $f\left(F_{j}\right)=0$ for $j \geq J$ (for some integer $J$ ), see [2, Proposition 11].

As we will see in the discussion below it might happen that $r_{j}(t)=0$ for finitely many $j$ and that we have then a similar infinite product representation for $\varphi(t)$. In any case the representation (4.2) is valid for sufficiently small $t$.

Set

$$
H_{k}(t):=\sum_{n<F_{k}} e^{i t f(n)}
$$

Then by defintion we have $H_{1}(t)=H_{2}(t)=1$ and

$$
H_{k}(t)=H_{k-1}(t)+e^{i t f\left(F_{k-1}\right)} H_{k-2}(t) \quad(k \geqslant 3) .
$$

Furthermore if we set

$$
A_{k}(t)=\left(\begin{array}{cc}
1 & e^{i t f\left(F_{k}\right)} \\
1 & 0
\end{array}\right)
$$

then (4.4) rewrites to

and consequently we have

$$
\left(\begin{array}{c}
H_{k}(t) \\
H_{k-1}(t)
\end{array}\right)=A_{k-1}(t)\left(\begin{array}{c}
H_{k-1}(t) \\
H_{k-2}(t)
\end{array}\right)
$$

$$
H_{k}(t)=\left(\begin{array}{ll}
1 & 0
\end{array}\right) A_{k-1}(t) A_{k-2}(t) \cdots A_{2}(t)\left(\begin{array}{l}
1 \\
1
\end{array}\right) .
$$


Next we assume that $r_{j}(t) \neq 0$ for all $j \geqslant 2$. We observe that the recurrence (4.3) is equivalent to the relation

which leads directly to

$$
A_{k}(t)\left(\begin{array}{c}
r_{k}(t) \\
1
\end{array}\right)=r_{k}(t)\left(\begin{array}{c}
r_{k+1}(t) \\
1
\end{array}\right)
$$

$$
H_{k}(t)=r_{2}(t) r_{3}(t) \cdots r_{k-1}(t) r_{k}(t)
$$

Thus, if we have a limiting distribution then the characteristic function of the limit is given

$$
\varphi(t)=\lim _{k \rightarrow \infty} \frac{1}{F_{k}} H_{k}(t)=\lim _{k \rightarrow \infty} \frac{\sqrt{5}}{\gamma^{k}} H_{k}(t)=\frac{\sqrt{5}}{\gamma} \prod_{j \geqslant 2} \frac{r_{j}(t)}{\gamma} .
$$

Next let us assume that $f\left(F_{k}\right) \rightarrow 0$ as $k \rightarrow \infty$. (This is certainly implied by (4.1).) By (4.4) this implies that for every fixed real number $t_{0}>0$ we have $r_{k}(t)=H_{k}(t) / H_{k-1}(t) \rightarrow \gamma$ uniformly for $|t| \leqslant t_{0}$. The converse is also true (here we have use again Lemma 9 of the Appendix). In particular this shows that for every fixed $t_{0}>0$ there exists $j_{0}$ such that $r_{j}(t) \neq 0$ for $|t| \leqslant t_{0}$ and $j \geqslant j_{0}$. In the same way as above we obtain

$$
\varphi(t)=\frac{\sqrt{5}}{\gamma^{j_{0}}} H_{j_{0}}(t) \prod_{j \geqslant j_{0}+1} \frac{r_{j}(t)}{\gamma} \quad\left(|t| \leqslant t_{0}\right),
$$

where $r_{j}(t)$ satisfies the same recurrence (4.3) as above for $j \geqslant j_{0}$, and where we can compute $r_{j_{0}+1}(t)$ by

$$
r_{j_{0}+1}(t)=\frac{H_{j_{0}+1}(t)}{H_{j_{0}}(t)} .
$$

Next we will study the recurrence (4.3) in more detail. For this purpose we use the following notation:

Then (4.3) rewrites to

$$
\eta_{k}=\eta_{k}(t):=e^{i t f\left(F_{k}\right)}-1, \quad \varepsilon_{k}=\varepsilon_{k}(t):=r_{k}(t)-\gamma .
$$

$$
\varepsilon_{k+1}=\frac{\eta_{k}-(\gamma-1) \varepsilon_{k}}{\gamma+\varepsilon_{k}}
$$

Note that - for notational simplicity - we skip the dependence on $t$. Further note that $r_{k}(t) \rightarrow \gamma$ is equivalent to $\varepsilon_{k} \rightarrow 0$.

In order to quantify the above considerations on the non-zeroness of $r_{k}(t)$ we note that $\left|\eta_{k}\right|=$ $\left|e^{i t f\left(F_{k}\right)}-1\right| \leqslant 2 \gamma-3$ and $\left|\varepsilon_{k}\right| \leqslant \gamma-1$ implies $\left|\gamma+\varepsilon_{k}\right| \geqslant 1$ and consequently

$$
\left|\varepsilon_{k+1}\right| \leqslant\left|\eta_{k}\right|+(\gamma-1)\left|\varepsilon_{k}\right| \leqslant 2 \gamma-3+(\gamma-1)^{2}=\gamma-1
$$

Lemma 6. Suppose that $f\left(F_{k}\right) \rightarrow 0$ as $k \rightarrow \infty$. Then the condition that the two series converge is equivalent to the statement that the two series

$$
\sum_{j \geqslant j_{0}} \varepsilon_{j} \text { and } \sum_{j \geqslant j_{0}}\left|\varepsilon_{j}\right|^{2}
$$

converge, where $j_{0}$ is chosen in a way that $\varepsilon_{j} \neq-\gamma$ for $j \geqslant j_{0}$.

Proof. Since $f\left(F_{j}\right) \rightarrow 0$ we also have that $\varepsilon_{j} \rightarrow 0$, and we can assume that $\left|t f\left(F_{j}\right)\right| \leqslant \pi$ and $\left|\varepsilon_{j}\right| \leqslant \frac{1}{2}$ for $j \geqslant j_{0}$. By using the inequalities $\frac{4}{\pi^{2}}|x| \leqslant\left|e^{i x}-1\right| \leqslant|x|$ (for $|x| \leqslant \pi$ ) it directly follows that

$$
\sum_{j \geqslant 0}\left|\eta_{j}\right|^{2}<\infty \text { if and only if } \sum_{j \geqslant j_{0}} f\left(F_{j}\right)^{2}<\infty .
$$

and by applying the expansion $e^{i x}-1=i x+O\left(x^{2}\right)$ (for $x \rightarrow 0$ ) it also follows that the series $\sum_{j} \eta_{j}$ is convergent if and only if the series $\sum_{j} f\left(F_{j}\right)$ is convergent.

Since $\left|\varepsilon_{j}\right| \leqslant \frac{1}{2}$ it follows that $\left|\gamma+\varepsilon_{j}\right| \geqslant \gamma-\frac{1}{2}$, and with $L=(\gamma-1) /\left(\gamma-\frac{1}{2}\right)<1$ we have

$$
\left|\varepsilon_{j}\right| \leqslant\left|\eta_{j-1}\right|+L\left|\varepsilon_{j-1}\right|
$$

and by iteration

$$
\left|\varepsilon_{j}\right| \leqslant \sum_{\ell=1}^{j-j_{0}}\left|\eta_{j-\ell}\right| L^{\ell-1}+L^{j-j_{0}}\left|\varepsilon_{j_{0}}\right|
$$


Consequently

$$
\begin{aligned}
\sum_{j \geqslant j_{0}}\left|\varepsilon_{j}\right|^{2} & \leqslant 2 \sum_{j \geqslant j_{0}} \sum_{k, \ell=1}^{j-j_{0}}\left|\eta_{j-\ell}\right|\left|\eta_{j-k}\right| L^{k+\ell-2}+2 \sum_{j \geqslant j_{0}} L^{2\left(j-j_{0}\right)}\left|\varepsilon_{j_{0}}\right|^{2} \\
& =2 \sum_{k, \ell \geqslant 1} L^{k+\ell-2} \sum_{j \geqslant j_{0}+\max \{k, \ell\}}\left|\eta_{j-\ell}\right|\left|\eta_{j-k}\right|+2 \frac{\left|\varepsilon_{j_{0}}\right|^{2}}{1-L^{2}} \\
& \leqslant \frac{1}{(1-L)^{2}} \sum_{j \geqslant j_{0}}\left|\eta_{j}\right|^{2}+2 \frac{\left|\varepsilon_{j_{0}}\right|^{2}}{1-L^{2}},
\end{aligned}
$$

where we have used the Cauchy-Schwarz inequality to derive

$$
\sum_{j \geqslant j_{0}+\max \{k, \ell\}}\left|\eta_{j-\ell}\right|\left|\eta_{j-k}\right| \leqslant \sum_{j \geqslant j_{0}}\left|\eta_{j}\right|^{2}
$$

Thus, if the series $\sum_{j}\left|\eta_{j}\right|^{2}$ is convergent, then the series $\sum_{j}\left|\varepsilon_{j}\right|^{2}$ converges, too.

The converse statement is much easier to prove. From (4.7) and the assumption $\left|\varepsilon_{j}\right| \leqslant \frac{1}{2}$ we obtain

and consequently

$$
\eta_{j}=\frac{\varepsilon_{j}}{\gamma}+\gamma \varepsilon_{j+1}+\varepsilon_{j} \varepsilon_{j+1}
$$

$$
\left|\eta_{j}\right| \leqslant c_{1}\left|\varepsilon_{j}\right|+c_{2}\left|\varepsilon_{j+1}\right|
$$

for some positive constants $c_{1}, c_{2}$. Hence, if the series $\sum_{j}\left|\varepsilon_{j}\right|^{2}$ is convergent, the same property holds for the series $\sum_{j}\left|\eta_{j}\right|^{2}$.

Next assume that the two series $\sum_{j} \eta_{j}$ and $\sum_{j}\left|\eta_{j}\right|^{2}$ converge. As argued above this implies that the series $\sum_{j}\left|\varepsilon_{j}\right|^{2}$ converges, too. By the Cauchy-Schwarz inequality this also implies that the series $\sum_{j} \varepsilon_{j} \varepsilon_{j+1}$ converges. Hence, by using the fact that $\varepsilon_{j} \rightarrow 0($ as $j \rightarrow \infty)$ and the relation (4.9) it follows that the sum

$$
\sum_{j=L}^{M} \varepsilon_{j}=\left(\frac{1}{\gamma}+\gamma\right)^{-1}\left(\sum_{j=L}^{M} \eta_{j}-\sum_{j=L}^{M} \varepsilon_{j} \varepsilon_{j+1}+\varepsilon_{L}-\varepsilon_{M+1}\right)
$$

can be made arbitrarily small and, thus, Cauchy's criterion implies the convergence of the series $\sum_{j} \varepsilon_{j}$

Again the converse implication is easier to obtain. If we assume that the two series $\sum_{j} \varepsilon_{j}$ and $\sum_{j}\left|\varepsilon_{j}\right|^{2}$ converge then by using (4.9) it directly follows that the series $\sum_{j} \eta_{j}$ converges. We just recall that the series $\sum_{j}\left|\eta_{j}\right|^{2}$ converges, too. Summing up this completes the proof of the lemma.

We are now in the position to prove Theorem 7. Suppose first that the two series (4.1) converge. Then by Lemma 6 the two series (4.8) converge, too, which implies that the infinite product

$$
\prod_{j \geqslant j_{0}+1} \frac{r_{j}(t)}{\gamma}=\prod_{j \geqslant j_{0}+1}\left(1+\frac{\varepsilon_{k}(t)}{\gamma}\right)
$$

converges. Thus, by (4.6) the limit

$$
\varphi(t)=\lim _{k \rightarrow \infty} \varphi_{F_{k}}(t)
$$

exists (where $\left.\varphi_{N}(t):=(1 / N) \sum_{n<N} \exp (i t f(n))\right)$. As in the $q$-adic case, this also implies that $\varphi_{N}(t) \rightarrow \varphi(t)$ (see Lemma 8). Finally, the limiting function $\varphi(t)$ is continuous at $t=0$ (for example, Lemma 7 implies that $\varphi(t)=1+O(t)$ as $t \rightarrow 0$ ) and the conclusion follows thanks to Lévy's theorem.

Now we assume that $f(n)$ has a limiting distribution. This implies that $H_{k}(t) / F_{k} \rightarrow \varphi(t)$, where $\varphi(t)$ is the characteristic function of the limiting distribution. By (4.4) this implies that $e^{i t f\left(F_{k}\right)} \rightarrow 1$ as $k \rightarrow \infty$ and consequently (also by using Lemma 9 in Appendix) $f\left(F_{k}\right) \rightarrow 0$ as $k \rightarrow \infty$. 
The convergence to $\varphi(t)$ can be also rewritten to

$$
\varphi(t)=\frac{\sqrt{5}}{\gamma} \lim _{k \rightarrow \infty} \frac{1}{\gamma^{k-1}}\left(\begin{array}{ll}
1 & 0
\end{array}\right) A_{k}(t) A_{k-1}(t) \cdots A_{2}(t)\left(\begin{array}{l}
1 \\
1
\end{array}\right) .
$$

Let $\|A\|_{2}:=\sqrt{\rho\left(A A^{*}\right)}$ denote the spectral norm of a matrix $A$ (here $\rho$ denotes the spectral radius and $A^{*}$ the Hermitian conjugate). It is easy to see that $\left\|A_{j}(t)\right\|_{2}=\gamma$. However we have $\left\|A_{j+1}(t) A_{j}(t)\right\|_{2} \leqslant \gamma^{2} \exp \left(-c\left|\eta_{j+1}\right|^{2}\right)$ for some constant $c>0$ since

$$
\begin{aligned}
A_{j+1}(t) A_{j}(t)\left(A_{j+1}(t) A_{j}(t)\right)^{*} & =\left(\begin{array}{cc}
5+\eta_{j+1}+\bar{\eta}_{j+1} & 3+\eta_{j+1} \\
3+\bar{\eta}_{j+1} & 2
\end{array}\right) \\
& =\left(\begin{array}{cc}
5-2\left(1-\cos \left(t f\left(F_{j+1}\right)\right)\right) & 3-\left(1-e^{i t f\left(F_{j+1}\right)}\right) \\
3-\left(1-e^{-i t f\left(F_{j+1}\right)}\right) & 2
\end{array}\right)
\end{aligned}
$$

and the spectral radius satisfies

$\rho\left(\left(\begin{array}{cc}5-2\left(1-\cos \left(t f\left(F_{j+1}\right)\right)\right) & 3-\left(1-e^{i t f\left(F_{j+1}\right)}\right) \\ 3-\left(1-e^{-i t f\left(F_{j+1}\right)}\right) & 2\end{array}\right)\right) \leqslant \gamma^{4}-c^{\prime}\left(1-\cos \left(t f\left(F_{j+1}\right)\right)\right) \leqslant \gamma^{4}-c^{\prime \prime}\left|\eta_{j+1}\right|^{2}$

for proper constants $c^{\prime}>0, c^{\prime \prime}>0$. Hence by taking norm at (4.10) and by grouping consecutive matrices together we obtain (similarly to the proof in the $q$-adic case) that

$$
\frac{1}{2} \leqslant|\varphi(t)| \leqslant \min \left\{\exp \left(-c \sum_{j \geqslant 1}\left\|\frac{t f\left(F_{2 j}\right)}{2 \pi}\right\|^{2}\right), \exp \left(-c \sum_{j \geqslant 1}\left\|\frac{t f\left(F_{2 j+1}\right)}{2 \pi}\right\|^{2}\right)\right\}
$$

which implies (as in the $q$-adic case) that the series $\sum_{j} f\left(F_{j}\right)^{2}$ converges. At this point we can now argue as in the proof of Lemma 6 and observe that the series $\sum_{j}\left|\varepsilon_{j}(t)\right|^{2}$ converges, too. We choose $t>0$ sufficiently small such that the representation (4.2) holds. Thus, the series $\sum_{j} \varepsilon_{j}(t)$ converges and consequently by Lemma 6 the two series (4.1) converge which completes the proof of Theorem 7 .

4.2. An Effective Version of the Zeckendorf Erdős-Wintner theorem. Similarly to Theorem 2 we can formulate a quantitative version for Zeckendorf additive functions that are, however, slightly weaker.

Theorem 8. Suppose that $f(n)$ is a real-valued Z-additive function such that the series

$$
\sum_{j \geqslant 2}\left|f\left(F_{j}\right)\right|
$$

converges. Set $L=\left\lceil\log _{\gamma}(\sqrt{5} N)\right\rceil$ and $h=\left\lceil\log _{\gamma}(T \log T)\right\rceil$. Then we have for all real numbers $T \geqslant 1$ such that $h \leqslant L / 2$

$$
\left\|F_{N}-F\right\|_{\infty} \ll Q_{F}(1 / T)+\frac{\log N}{T}+T \sum_{j>L-2 h+1}\left|f\left(F_{j}\right)\right|
$$

where $F_{N}$ denotes the distribution function of $(f(n): n<N)$ and $F$ the limiting distribution function.

We note that there is also an analogue to Theorem 3 that is of the form

$$
\begin{aligned}
\left\|F_{N}-F\right\|_{\infty} & \ll \frac{1}{T} \int_{0}^{T} \exp \left(-c_{2} t^{2} \sum_{j \in \tilde{S}(t)} f\left(F_{j}\right)^{2}\right) \mathrm{d} t+\frac{\log N}{T}+T \sum_{j=L-2 h+1}^{L}\left|f\left(F_{j}\right)\right| \\
& +\int_{1 / T}^{T} \min \left\{\frac{1}{1+t}, \sum_{j \geqslant L-h}\left|f\left(F_{j}\right)\right|\right\} \exp \left(-c_{2} t^{2} \sum_{j \in \tilde{S}(t), j<L-h} f\left(F_{j}\right)^{2}\right) \mathrm{d} t,
\end{aligned}
$$

where

and $c_{2}>0$ is some constant.

$$
\tilde{S}(t)=\left\{j \geqslant 2:\left|f\left(F_{j}\right)\right| \leqslant \pi /|t|\right\}
$$

Remark 1. By choosing $h=\left\lceil\log _{\gamma}(T \log N \log T)\right\rceil$ in the two previous upper bounds, the term $\log (N) / T$ disappears. 
The main differences between Theorems 2 and 3 and Theorem 8 are the additional term $(\log N) / T$ and the need of the assumption (4.11), at least for the upper bound (4.12) (the upper bound (4.13) works in principle in all cases). Both seem to be artefacts of the proof, however, it seems that the current proof methods are not strong enough to overcome these artefacts. Nevertheless, the proof uses quite the same ideas as that of the previous theorem. The main technical difficulty is to handle non-commutative matrix products.

We start with two lemmas that are analogues to Lemma 2 and to Lemma 3.

Lemma 7. Let $f(n)$ be a real-valued Z-additive function such that the two series (4.1) converge. Then we have

$$
\frac{1}{N} \sum_{n<N}|f(n)|=O(1)
$$

as $N \rightarrow \infty$.

Proof. We choose $F_{L}$ such that $F_{L-1}<N \leqslant F_{L}$ and will prove that

$$
\sum_{n<F_{L}}|f(n)|=O\left(F_{L}\right)
$$

Clearly this implies (4.14).

For this purpose we consider first the sums

$$
S_{k}:=\sum_{n<F_{k}} f(n)
$$

By definition of $f$ we obtain the recurrence

$$
\begin{aligned}
S_{k} & =\sum_{n<F_{k-1}} f(n)+\sum_{n^{\prime}<F_{k-2}} f\left(F_{k-1}+n^{\prime}\right) \\
& =S_{k-1}+S_{k-2}+F_{k-2} f\left(F_{k-1}\right)
\end{aligned}
$$

which leads (by induction) to

$$
S_{k}=\sum_{\ell=2}^{k-1} F_{k-\ell} F_{\ell-1} f\left(F_{\ell}\right)
$$

The representation can be used to prove $S_{k}=O\left(F_{k}\right)$ but we will (4.15) directly.

Similarly we can treat the sum of squares. From the recurrence

$$
\begin{aligned}
T_{k} & :=\sum_{n<F_{k}} f(n)^{2}=\sum_{n<F_{k-1}} f(n)^{2}+\sum_{n^{\prime}<F_{k-2}} f\left(F_{k-1}+n^{\prime}\right)^{2} \\
& =S_{k-1}+S_{k-2}+F_{k-2} f\left(F_{k-1}\right)^{2}+2 S_{k-2} f\left(F_{k-1}\right)
\end{aligned}
$$

we obtain (again) by induction

$$
T_{k}=\sum_{\ell=2}^{k-1} F_{k-\ell} F_{\ell-1} f\left(F_{\ell}\right)^{2}+2 \sum_{\ell=2}^{k-1} F_{k-\ell} S_{\ell-1} f\left(F_{\ell}\right)
$$

The first sum can be easily handled:

$$
\sum_{\ell=2}^{k-1} F_{k-\ell} F_{\ell-1} f\left(F_{\ell}\right)^{2}=O\left(\gamma^{k-1} \sum_{\ell=2}^{k-1} f\left(F_{\ell}\right)^{2}\right)=O\left(F_{k}\right) .
$$


For the second sum we use (4.15) and obtain

$$
\begin{aligned}
2 \sum_{\ell=2}^{k-1} F_{k-\ell} & S_{\ell-1} f\left(F_{\ell}\right)=2 \sum_{\ell=2}^{k-1} \sum_{j=2}^{\ell-2} F_{k-\ell} F_{\ell-j-1} F_{j-1} f\left(F_{\ell}\right) f\left(F_{j}\right) \\
& =\frac{2}{5 \sqrt{5}} \sum_{\ell=2}^{k-1} \sum_{j=2}^{\ell-2}\left(\gamma^{k-\ell}+(-1)^{k-\ell+1} \gamma^{\ell-k}\right) \\
& =2 \frac{\gamma^{k-2}}{5 \sqrt{5}} \sum_{\ell=2}^{k-1} \sum_{j=2}^{\ell-2} f\left(\gamma_{\ell}\right) f\left(F_{j}\right)+\frac{2}{5 \sqrt{5}} \sum_{\ell=2}^{k-1} \sum_{j=2}^{\ell-2} \gamma^{k-2 j}(-1)^{j} f\left(F_{\ell}\right) f\left(F_{j}\right) \\
& +\frac{2}{5 \sqrt{5}} \sum_{\ell=2}^{k-1} \sum_{j=2}^{\ell-2} \gamma^{k-2 \ell+2 j}(-1)^{\ell-j} f\left(F_{\ell}\right) f\left(F_{j}\right)+\frac{2}{5 \sqrt{5}} \sum_{\ell=2}^{k-1} \sum_{j=2}^{\ell-2} \gamma^{2 \ell-k-2}(-1)^{k-\ell+1} f\left(F_{\ell}\right) f\left(F_{j}\right) \\
& +\frac{2}{5 \sqrt{5}} \sum_{\ell=2}^{k-1} \sum_{j=2}^{\ell-2} \gamma^{k-2 \ell+2}(-1)^{\ell} f\left(F_{\ell}\right) f\left(F_{j}\right)+\frac{2}{5 \sqrt{5}} \sum_{\ell=2}^{k-1} \sum_{j=2}^{\ell-2} \gamma^{2 \ell-2 j-k}(-1)^{k-\ell+j+1} f\left(F_{\ell}\right) f\left(F_{j}\right) \\
& +\frac{2}{5 \sqrt{5}} \sum_{\ell=2}^{k-1} \sum_{j=2}^{\ell-2} \gamma^{2 j-k}(-1)^{k-j+1} f\left(F_{\ell}\right) f\left(F_{j}\right)+\frac{2}{5 \sqrt{5}} \sum_{\ell=2}^{k-1} \sum_{j=2}^{\ell-2} \gamma^{2-k}(-1)^{k+1} f\left(F_{\ell}\right) f\left(F_{j}\right) .
\end{aligned}
$$

Since

$$
R:=\sum_{\ell=2}^{k-1} \sum_{j=2}^{\ell-2} f\left(F_{\ell}\right) f\left(F_{j}\right)=\frac{1}{2}\left(\sum_{j=2}^{k-1} f\left(F_{j}\right)\right)^{2}-\frac{1}{2} \sum_{j=2}^{k-1} f\left(F_{j}\right)^{2}-\sum_{j=3}^{k-1} f\left(F_{j}\right) f\left(F_{j-1}\right)
$$

and

$$
\left|\sum_{j=3}^{k-1} f\left(F_{j}\right) f\left(F_{j-1}\right)\right| \leqslant\left(\sum_{j=3}^{k-1} f\left(F_{j}\right)^{2} \cdot \sum_{j=3}^{k-1} f\left(F_{j-1}\right)^{2}\right)^{1 / 2}=O(1)
$$

it follows that $R=O(1)$ and, thus, the first part of the sum is of order $O\left(F_{k}\right)$. The other parts can be handled even more directly. For the sake of shortness we only discuss the next three terms (that are also asymptotically most significant). The remaining four terms are really easy to bound. First we have

$$
\begin{aligned}
\sum_{\ell=2}^{k-1} \sum_{j=2}^{\ell-2} \gamma^{k-2 j}(-1)^{j} f\left(F_{\ell}\right) f\left(F_{j}\right) & =\sum_{j=2}^{k-3} \gamma^{k-2 j}(-1)^{j} f\left(F_{j}\right) \sum_{\ell=j+2}^{k-1} f\left(F_{\ell}\right) \\
& =\sum_{j=2}^{k-3} \gamma^{k-2 j}\left|f\left(F_{j}\right)\right| O(1) \\
& =O\left(\left(\sum_{j=2}^{k-3} \gamma^{2 k-4 j} \sum_{j=2}^{k-3} f\left(F_{j}\right)^{2}\right)^{1 / 2}\right) \\
& =O\left(F_{k}\right) .
\end{aligned}
$$

Second, we obtains

$$
\begin{aligned}
\sum_{\ell=2}^{k-1} \sum_{j=2}^{\ell-2} \gamma^{k-2 \ell+2 j}(-1)^{\ell-j} f\left(F_{\ell}\right) f\left(F_{j}\right) & =\sum_{r=2}^{k-3} \gamma^{k-2 r}(-1)^{r} \sum_{j=2}^{k-r-1} f\left(F_{j+r}\right) f\left(F_{j}\right) \\
& =O\left(\sum_{r=2}^{k-3} \gamma^{k-2 r} \sum_{j=2}^{k-1} f\left(F_{j}\right)^{2}\right) \\
& =O\left(F_{k}\right)
\end{aligned}
$$


And third, we get

$$
\begin{aligned}
\sum_{\ell=2}^{k-1} \sum_{j=2}^{\ell-2} \gamma^{2 \ell-k-2}(-1)^{k-\ell+1} f\left(F_{\ell}\right) f\left(F_{j}\right) & =\sum_{\ell=2}^{k-1} \gamma^{2 \ell-k-2}(-1)^{k-\ell+1} f\left(F_{\ell}\right) \sum_{j=2}^{\ell-2} f\left(F_{j}\right) \\
& =\sum_{\ell=2}^{k-1} \gamma^{2 \ell-k-2}\left|f\left(F_{\ell}\right)\right| O(1) \\
& =O\left(\left(\sum_{\ell=2}^{k-1} \gamma^{2 k-4 \ell} \sum_{\ell=2}^{k-1} f\left(F_{\ell}\right)^{2}\right)^{1 / 2}\right) \\
& =O\left(F_{k}\right) .
\end{aligned}
$$

Finally by Cauchy-Schwarz's inequality we obtain

$$
\sum_{n<F_{L}}|f(n)| \leqslant F_{L}^{1 / 2}\left(\sum_{n<F_{L}} f(n)^{2}\right)^{1 / 2}=O\left(\left(F_{L} T_{L}\right)^{1 / 2}\right)=O\left(F_{L}\right)
$$

as required. This completes the proof of the lemma.

Lemma 8. Suppose that $f(n)$ is real-valued and $Z$-additive. Then we have for all integers $1 \leqslant h \leqslant$ $L / 2$

$$
\left|\varphi_{N}(t)-\varphi_{F_{L}}(t)\right| \leqslant C_{1} \frac{\log N}{\gamma^{h}}+C_{2} \sum_{j=L-2 h+1}^{L}\left(1-\cos \left(t f\left(F_{j}\right)\right)^{1 / 2}\right.
$$

where $L=\left\lceil\log _{\gamma}(\sqrt{5} N)\right\rceil$ and $C_{1}$ and $C_{2}$ are two absolute positive constants.

Proof. For $r \geqslant 2$ we set

$$
f_{r}(n):=\prod_{j=2}^{r-1} f\left(\delta_{j}(n) F_{j}\right)
$$

and

$$
\varphi_{N}^{(r)}(t):=\frac{1}{N} \sum_{n<N} e^{i t f_{r}(n)}
$$

The difference $\varphi_{N}(t)-\varphi_{F_{L}}(t)$ is now estimated in the following way:

$$
\begin{aligned}
\left|\varphi_{N}(t)-\varphi_{F_{L}}(t)\right| & \leqslant\left|\varphi_{N}(t)-\varphi_{N}^{(L-h)}(t)\right| \\
& +\left|\varphi_{N}^{(L-h)}(t)-\frac{F_{L-h-1}}{\gamma^{L-h-2}} \varphi_{F_{L-h-1}}(t)-\frac{F_{L-h-2}}{\gamma^{L-h-1}} e^{i t f\left(F_{L-h-1}\right)} \varphi_{F_{L-h-2}}(t)\right| \\
& +\frac{F_{L-h-1}}{\gamma^{L-h-2}}\left|\varphi_{F_{L-h-1}}(t)-\varphi_{F_{L}}(t)\right|+\frac{F_{L-h-1}}{\gamma^{L-h-2}}\left|\varphi_{F_{L-h-2}}(t)-\varphi_{F_{L}}(t)\right| .
\end{aligned}
$$

Note that $F_{L-h-1} / \gamma^{L-h-2}+F_{L-h-2} / \gamma^{L-h-1}=1$.

We consider now the four parts on the right hand side of (4.16) separately. For the first part we observe that $f(n)=f_{L-h}(n)$ if $n<F_{L-h}$. If $n \geqslant F_{L-h}$ then we have

$$
\begin{aligned}
\left|e^{i t f(n)}-e^{i t f_{L-h}(n)}\right| & =\left|\prod_{j \geqslant L-h} e^{i t f\left(\delta_{j}(n) F_{j}\right)}-1\right| \\
& \leqslant \sum_{j=L-h}^{L}\left|e^{i t f\left(\delta_{j}(n) F_{j}\right)}-1\right| \\
& =\sqrt{2} \sum_{j=L-h}^{L} \sqrt{1-\cos \left(t f\left(\delta_{j}(n) F_{j}\right)\right)}
\end{aligned}
$$


and consequently

$$
\left|\varphi_{N}(t)-\varphi_{N}^{(L-h)}(t)\right| \leqslant \sqrt{2} \sum_{j=L-h}^{L} \sqrt{1-\cos \left(t f\left(F_{j}\right)\right)} .
$$

Next we use the fact (see [28]) that for every $r \geqslant 2$ there exists a partition of $[0,1)$ into $F_{r}$ intervals $I_{r}(k), 0 \leqslant k<F_{r}$, of lengths $\left|I_{r}(k)\right|=\gamma^{2-r}$ if $0 \leqslant k<F_{r-1}$ and $\left|I_{r}(k)\right|=\gamma^{1-r}$ if $F_{r-1} \leqslant k<F_{r}$ such that

$$
\{n \gamma\} \in I_{r}(k) \quad \text { if and only if } \quad\left(\delta_{2}(n), \delta_{3}(n), \ldots, \delta_{r-1}(n)\right)=\left(\delta_{2}(k), \delta_{3}(k), \ldots, \delta_{r-1}(k)\right) .
$$

Since the discrepancy of the sequence $\{n \gamma\}$ is of order $(\log N) / N$ we, thus, obtain

$$
\begin{aligned}
\varphi_{N}^{(r)}(t) & =\sum_{k<F_{r}} \frac{\#\left\{n<N:\{n \gamma\} \in I_{r}(k)\right\}}{N} e^{i t f(k)} \\
& =\sum_{k<F_{r}}\left(\left|I_{r}(k)\right|+O\left(\frac{\log N}{N}\right)\right) e^{i t f(k)} \\
& =\sum_{k<F_{r}}\left|I_{r}(k)\right| e^{i t f(k)}+O\left(\frac{F_{r} \log N}{N}\right) .
\end{aligned}
$$

Clearly we have

$$
\sum_{k<F_{r}}\left|I_{r}(k)\right| e^{i t f(k)}=\frac{F_{r-1}}{\gamma^{r-2}} \varphi_{F_{r-1}}(t)+\frac{F_{r-2}}{\gamma^{r-1}} e^{i t f\left(F_{r-1}\right)} \varphi_{F_{r-2}}(t)
$$

which implies (by setting $r=L-h$ )

$$
\left|\varphi_{N}^{(L-h)}(t)-\frac{F_{L-h-1}}{\gamma^{L-h-2}} \varphi_{F_{L-h-1}}(t)-\frac{F_{L-h-2}}{\gamma^{L-h-1}} e^{i t f\left(F_{L-h-1}\right)} \varphi_{F_{L-h-2}}(t)\right| \leqslant C \frac{\log N}{\gamma^{h}}
$$

for some constant $C>0$.

For the final part we recall the defintion (4.5) of the matrices $A_{k}=A_{k}(t)$. We further set

$$
A:=\left(\begin{array}{ll}
1 & 1 \\
1 & 0
\end{array}\right)
$$

and note that $\left\|A-A_{k}\right\|_{2}=\left|\eta_{k}\right|$ and $\left\|A_{k}\right\|_{2}=\|A\|_{2}=\gamma$. For exmaple, we have

$$
\left\|A^{h}-A_{L} A_{L-1} \cdots A_{L-h+1}\right\|_{2} \leqslant \gamma^{h-1} \sum_{j=L-h+1}^{L}\left\|A-A_{j}\right\|_{2}=\gamma^{h-1} \sum_{j=L-h+1}^{L}\left|\eta_{j}\right| .
$$

Note also that $A^{h}=\gamma^{h} M+O\left(\gamma^{-h}\right)$ where $M$ is the matrix that projects to the direction $(\gamma, 1)$ and satisfies $M^{2}=M$. By using the relation $F_{L} / F_{L-h}=\gamma^{h}+O\left(\gamma^{3 h-2 L}\right)$ and the decomposition

$$
\begin{aligned}
A_{L} A_{L-1} \cdots A_{2}- & \frac{F_{L}}{F_{L-h}} A_{L-h} A_{L-h-1} \cdots A_{2}=A_{L} A_{L-1} \cdots A_{2}-\gamma^{h} A_{L-h} A_{L-h-1} \cdots A_{2}+O\left(\gamma^{2 h-L}\right) \\
& =\left(A_{L} A_{L-1} \cdots A_{L-h+1}-A^{h}\right) A_{L-h} A_{L-h-1} \cdots A_{2} \\
& +\left(A^{h}-\gamma^{h} I\right) A_{L-h} A_{L-h-1} \cdots A_{2}+O\left(\gamma^{2 h-L}\right) \\
& =\left(A_{L} A_{L-1} \cdots A_{L-h+1}-A^{h}\right) A_{L-h} A_{L-h-1} \cdots A_{2} \\
& +\gamma^{h}(M-I) A_{L-h} A_{L-h-1} \cdots A_{2}+O\left(\gamma^{2 h-L}\right)+O\left(\gamma^{L-2 h}\right) \\
& =\left(A_{L} A_{L-1} \cdots A_{L-h+1}-A^{h}\right) A_{L-h} A_{L-h-1} \cdots A_{2} \\
& +\gamma^{h}(M-I)\left(A_{L-h} A_{L-h-1} \cdots A_{L-2 h+1}-A^{h}\right) A_{L-2 h} \cdots A_{2} \\
& +\gamma^{h}(M-I) \gamma^{h} M A_{L-2 h} \cdots A_{2}+O\left(\gamma^{L-2 h}\right)
\end{aligned}
$$


(where $I$ is the $2 \times 2$ identity matrix) and by noting again that $(M-I) M=0$, we, thus, obtain

$$
\begin{aligned}
\left\|A_{L} A_{L-1} \cdots A_{2}-\frac{F_{L}}{F_{L-h}} A_{L-h} A_{L-h-1} \cdots A_{2}\right\|_{2} & \leqslant \gamma^{L-2} \sum_{j=L-h+1}^{L}\left|\eta_{j}\right| \\
& +\gamma^{L-2} \sum_{j=L-2 h+1}^{L-h}\left|\eta_{j}\right|+O\left(\gamma^{L-2 h}\right)
\end{aligned}
$$

and consequently

$$
\begin{aligned}
\left|\varphi_{F_{L}}(t)-\varphi_{F_{L-h}}(t)\right| & \leqslant \sqrt{2} \frac{\gamma^{L-2}}{F_{L}} \sum_{j=L-2 h+1}^{L}\left|\eta_{j}\right|+O\left(\gamma^{-2 h}\right) \\
& \ll \sum_{j=L-2 h+1}^{L} \sqrt{1-\cos \left(t f\left(F_{j}\right)\right)}+\gamma^{-2 h} .
\end{aligned}
$$

The same estimate holds if we replace $h$ by $h+1$ or $h+2$.

Summing up, this completes the proof of the lemma.

We are now ready to prove Theorem 8 that runs along the same lines as the proof of Theorems 2 and 3, in particular we use (again) the Berry-Esseen inequality. Instead of the upper bound (2.16) we get (with the help of the representation (4.10) and the bound $\left\|A_{j}(t) A_{j+1}(t)\right\|_{2} \leqslant \gamma^{2} \exp \left(-c\left|\eta_{j}\right|^{2}\right)$ ) the estimate

$$
|\varphi(t)| \leqslant \exp \left(-c_{1}^{\prime} t^{2} \sum_{j \in \tilde{S}(t)} f\left(F_{j}\right)^{2}\right)
$$

which leads to an upper bound for $Q_{F}(1 / T)$.

From Lemma 7 we get $\varphi(t)-\varphi_{N}(t)=O(|t|)$ which can be used to estimate the integral $\int_{-1 / T}^{1 / T} \frac{1}{|t|}\left|\varphi(t)-\varphi_{N}(t)\right| \mathrm{d} t$.

The integral $\int_{1 / T \leqslant|t| \leqslant T} \frac{1}{|t|}\left|\varphi(t)-\varphi_{N}(t)\right| \mathrm{d} t$ is (again) split into two parts:

$$
\int_{1 / T \leqslant|t| \leqslant T} \frac{\left|\varphi(t)-\varphi_{F_{L}}(t)\right|}{|t|} \mathrm{d} t+\int_{1 / T \leqslant|t| \leqslant T} \frac{\left|\varphi_{F_{L}}(t)-\varphi_{N}(t)\right|}{|t|} \mathrm{d} t .
$$

For the second part we apply Lemma 8. Note that we choose $h \leqslant L / 2$ in a way that $\log T \log N / \gamma^{h} \ll 1 / T$. For the first part we need proper bounds for the difference $\varphi(t)-\varphi_{F_{L}}(t)$. For $t \rightarrow 0$ we certainly have

$$
\begin{aligned}
\left|\varphi(t)-\varphi_{F_{L}}(t)\right| & =\left|\varphi_{F_{L}}(t)\right| 1-\frac{\varphi(t)}{\varphi_{F_{L}}(t)} \mid \\
& \ll|t| \exp \left(-c_{1}^{\prime} t^{2} \sum_{j<L, j \in \tilde{S}(t)} f\left(F_{j}\right)^{2}\right) .
\end{aligned}
$$

By using the matrix product representation we also obtain (for all $t$ )

$$
\left|\varphi(t)-\varphi_{F_{L}}(t)\right| \ll \exp \left(-c_{1}^{\prime} t^{2} \sum_{j<L, j \in \tilde{S}(t)} f\left(F_{j}\right)^{2}\right) .
$$

Finally by using the same methods as in the proof of Lemma 8 we get

$$
\begin{aligned}
\left|\varphi(t)-\varphi_{F_{L}}(t)\right| & \ll \sum_{j \geqslant L-h}\left|\eta_{j}\right| \cdot \gamma^{h-L}\left\|\prod_{j=2}^{L-h-1} A_{j}(t)\right\| \|_{2}+O\left(\gamma^{-2 h}\right) \\
& \ll|t| \sum_{j \geqslant L-h}\left|f\left(F_{j}\right)\right| \exp \left(-c_{1}^{\prime} t^{2} \sum_{j<L-h, j \in \tilde{S}(t)} f\left(F_{j}\right)^{2}\right)+O\left(\gamma^{-2 h}\right)
\end{aligned}
$$


Hence, we obtain

$$
\begin{aligned}
\int_{1 / T \leqslant|t| \leqslant T} \frac{\left|\varphi(t)-\varphi_{F_{L}}(t)\right|}{|t|} \mathrm{d} t & \ll \int_{1 / T}^{T} \min \left\{\frac{1}{1+t}, \sum_{j \geqslant L-h}\left|f\left(F_{j}\right)\right|\right\} \exp \left(-c_{2} t^{2} \sum_{j \in \tilde{S}(t), j<L-h} f\left(F_{j}\right)^{2}\right) \mathrm{d} t \\
& +\frac{\log T}{\gamma^{2 h}} .
\end{aligned}
$$

Note that $\log T / \gamma^{2 h} \ll 1 / T$ and so we are done.

Example. Again we consider the most easy example (similarly to the $q$-ary case). We suppose that

$$
c_{1} j^{-\alpha} \leqslant f\left(F_{j}\right) \leqslant c_{2} j^{-\alpha}
$$

for $j \geqslant 2$ and some $\alpha>1$, where $c_{1}, c_{2}$ are positive constants. By applying Theorem 8 , choosing $h$ depending on $N$ as in Remark 1 and by calculations that are very similiar to the $q$-ary case we obtain

$$
\left\|F-F_{N}\right\|_{\infty} \ll\left\{\begin{array}{cl}
(\log N)^{1-\alpha} & \text { for } 1<\alpha<2 \\
\sqrt{\log \log N(\log N)^{\frac{\alpha}{2}}} & \text { for } \alpha \geq 2
\end{array}\right.
$$

Remark. They are open questions how improve Theorem 8 and how far Theorems 7 and 8 can be generalized to base sequences $G_{n}$ that satisfy linear recurrences with constant coefficients. It should be certainly feasible to handle base sequences $\left(G_{n}\right)$ that are given by $G_{0}=1, G_{1}=a$ and $G_{n+2}=a G_{n+1}+G_{n}(n \geqslant 0)$, where $a \geqslant 1$ is a given integer, but even this case seems to be very involved.

Acknowledgment. The authors are very grateful to Lukas Spiegelhofer and to Gérald Tenenbaum for their valuable comments to a previous version of this manuscript.

\section{REFERENCES}

[1] G. Barat And P. J. Grabner, Disitribution properties of G-additive functions, Journal of Number Theory, 60 (1996), pp. 103-123.

[2] G. Barat And P. J. Grabner, Limit distribution of Q-additive functions from an ergodic point of view., Ann. Univ. Sci. Budap. Rolando Eötvös, Sect. Comput., 28 (2008), pp. 55-78.

[3] D. BerEnd AND G. Kolesnik, Joint distribution of completely q-additive functions in residue classes, Journal of Number Theory, 160 (2016), pp. $716-738$.

[4] I. Berkes, On the asymptotic behaviour of $\sum f\left(n_{k} x\right)$. I. Main Theorems, Z. Wahr. verv. Geb., 34 (1976), pp. 319-345.

[5] J. Coquet, Sur les fonctions S-multiplicatives et S-additives, Thèse de doctorat de Troisième Cycle, Université Paris-Sud, Orsay, (1975).

[6] C. Dartyge and G. Tenenbaum, Sommes des chiffres de multiples d'entiers., Ann. Inst. Fourier, 55 (2005), pp. $2423-2474$

[7] - Congruences de sommes de chiffres de valeurs polynomiales., Bull. Lond. Math. Soc., 38 (2006), pp. 6169.

[8] H. Delange, Sur les fonctions q-additives ou q-multiplicatives, Acta Arithmetica 21, (1972), pp. 285-298.

[9] M. Drmota, The joint distribution of q-additive functions, Acta Arithmetica, 100 (2001), pp. 17-39.

[10] M. Drmota and P. J. Grabner, Analysis of digital functions and applications., in Combinatorics, automata, and number theory., Cambridge: Cambridge University Press, 2010, pp. 452-504.

[11] M. Drmota And G. Gutenbrunner, The joint distribution of Q-additive functions on polynomials over finite fields, Journal de Théorie des Nombres de Bordeaux, 17 (2005), pp. 125-150.

[12] P. D. T. A. Elliote, Probalistic Number Theory, I : mean value theorems, Grundlehren der Math. Wiss. 239, Springer-Verlag, New York, Berlin, Heidelberg, 1979.

[13] P. Erdős, On the density of some sequences of numbers. I., J. Lond. Math. Soc., 10 (1935), pp. 120-125.

[14] - On the density of some sequences of numbers. II., J. Lond. Math. Soc., 12 (1937), pp. 7-11.

[15] P. ERdős, On a family of symmetric Bernoulli convolutions, Amer. J. Math, 61 (1939).

[16] P. Erdős And A. Wintner, Additive arithmetical functions and statistical independence, Amer. J. Math., 61 (1939), pp. 713-721.

[17] P. ERDős, On the density of some sequences of numbers. III., J. Lond. Math. Soc., 13 (1938), pp. 119-127.

[18] A. Fan And J. Konieczny, On uniformity of q-multiplicative sequences., Bull. Lond. Math. Soc., 51 (2019), pp. $466-488$.

[19] A. Haddley, P. Lertchoosakul, And R. NAIR, The Halton sequence and its discrepancy in the Cantor expansion, Periodica Mathematica Hungarica, 75 (1), (2017), pp. $128-141$.

[20] P. Hartman, The divergence of non-harmonic gap series, Duke Math. J., 9 (1942), pp. $404-405$. 
[21] I. KÁtAI, On q-additive and q-multiplicative functions, in Number Theory and Discrete Mathematics, A. K. Agarwal, B. C. Berndt, C. F. Krattenthaler, G. L. Mullen, K. Ramachandra, and M. Waldschmidt, eds., Basel, 2002, Birkhäuser Basel, pp. 61-76.

[22] M. MADRITSCH, The summatory function of q-additive functions on pseudo-polynomial sequences, Journal de Théorie des Nombres de Bordeaux, 24 (2012), pp. 153-171.

[23] B. Martin, C. Mauduit, and J. Rivat, Fonctions digitales le long des nombres premiers., Acta Arith., 170 (2015), pp. 175-197.

[24] C. Mauduit and J. Rivat, Propriétés q-multiplicatives de la suite $\left\lfloor n^{c}\right\rfloor, c>1 .$, Acta Arith., 118 (2005), pp. $187-203$.

[25] — La somme des chiffres des carrés., Acta Math., 203 (2009), pp. 107-148.

[26] - Sur un problème de Gelfond: la somme des chiffres des nombres premiers., Ann. Math. (2), 171 (2010), pp. 1591-1646.

[27] Y. Peres, W. Schlag, And B. Solomyak, Sixty years of Bernoulli convolutions, Birkhäuser Basel, Basel, 2000.

[28] L. SpIEgelhofer, Correlations for numeration systems, PhD thesis, TU Wien and Aix-Marseille Université, 2014.

[29] S. TAKAhashi, An asymptotic property of a gap sequence, Proc. Japan Acad., 38 (1962), pp. $101-104$.

[30] G. Tenenbaum, Sur la non-dérivabilité de fonctions périodiques associées à certaines formules sommatoires., in The mathematics of Paul Erdös. Vol. I, Berlin: Springer, 1997, pp. 117-128.

[31] - Introduction to analytic and probabilistic number theory. Transl. from the 3rd French edition by Patrick D. F. Ion. 3rd expanded ed., vol. 163, Providence, RI: American Mathematical Society (AMS), 3rd expanded ed. ed., 2015.

[32] G. Tenenbaum and J. Verwee, Effective Erdős-Wintner theorems, 2020.

\section{Appendix A}

The following property is probably well known, however, we could not find a proper reference. We thank Lukas Spiegelhofer (TU Wien) and Gérald Tenenbaum (Nancy) for the following two nice proofs.

Lemma 9. Let be $\eta>0$ and let $\left(a_{n}\right)_{n}$ be a sequence of real numbers such that, for all $\tau \in(0, \eta]$

$$
\left\|\tau a_{n}\right\| \rightarrow 0
$$

where $\|\cdot\|$ is the distance to nearest integer function. Then $a_{n} \rightarrow 0$.

1st Proof (Spiegelhofer). By contradiction, we assume that $a_{n}$ does not converge to 0 . This means that there exist $\varepsilon>0$ and an infinite set $I$ of natural integers such that $\left|a_{n}\right|>\varepsilon$ for $n \in I$. Without loss of generality we can asssume that $\varepsilon \leqslant 1 / \eta$.

We define the following sets

$$
A_{n}:=\left\{\tau \in(0, \eta):\left\|\tau a_{n}\right\|>\frac{\eta \varepsilon}{6}\right\} .
$$

If $n \in I$ we observe that $\Lambda\left(A_{n}\right) \geqslant \eta / 3$, where $\Lambda$ denotes the Lebesgue measure. We just have to remark that $a_{n} \cdot A_{n}$ results from $a_{n} \cdot(0, \eta)$ by cutting out $\leqslant a_{n} \cdot \eta+1$ many intervals of length less or equal than $\eta \varepsilon / 3 \leqslant 1 / 3$. Thus,

$$
\Lambda\left(A_{n}\right) \geqslant \eta-\left(\eta+\frac{1}{a_{n}}\right) \frac{\eta \varepsilon}{3} \geqslant \eta-\frac{2 \eta}{3}=\frac{\eta}{3} .
$$

Let us now consider the following sets

$$
B_{m}:=\left\{\tau \in(0, \eta]: \forall n \geqslant m, \tau \notin A_{n}\right\}=(0, \eta] \backslash \bigcup_{n \geqslant m} A_{n}
$$

Note that $B_{m} \subseteq B_{m+1}$. Since $\left\|\tau a_{n}\right\| \rightarrow 0$ it follows that there exists $m=m(\tau)$ such that $\tau \in B_{m}$. Hence, $\bigcup_{m=0}^{\infty} B_{m}=(0, \eta]$. Consequently the continuity of the Lebesgue measure implies

$$
\lim _{m \rightarrow \infty} \Lambda\left(B_{m}\right)=\Lambda\left(\bigcup_{m=0}^{\infty} B_{m}\right)=\Lambda((0, \eta])=\eta .
$$

However, for all $m \geqslant 0$, there exists $n \geqslant m$ with $n \in I$. By definition $B_{n} \subseteq(0, \eta] \backslash A_{n}$, which implies $\Lambda\left(B_{m}\right) \leqslant \eta-\Lambda\left(A_{n}\right) \leqslant 2 \eta / 3$, whence the contradiction to (4.17). 
2nd Proof (Tenenbaum). By assumption $\left\|\tau a_{n}\right\| \rightarrow 0$ (for $\tau \in(0, \eta]$ ). Hence, the functions $f_{n}(\tau)=$ $1-\cos \left(2 \pi \tau a_{n}\right)$ converges pointwise to 0 on this interval. By Lebesgue's theorem it follows that, as $n \rightarrow \infty$,

$$
\frac{1}{\eta} \int_{0}^{\eta} f_{n}(\tau) d \tau=1-\frac{\sin \left(2 \pi \eta a_{n}\right)}{2 \pi \eta a_{n}} \rightarrow 0
$$

Thus, the sequence $\left(a_{n}\right)_{n}$ has to be bounded. Consequently, if $\tau>0$ is sufficiently small we have $\left\|\tau a_{n}\right\|=\left|\tau a_{n}\right|$ which implies that that $\left|\tau a_{n}\right| \rightarrow 0$. Of course this also proves $a_{n} \rightarrow 0$ as proposed.

\section{ApPEndix B}

In Theorem 4 an explicit value for $c(\beta)$ is not given. However, Gérald Tenenbaum mentioned to us that one can use, for example, the following explicit bound

$$
\left\|F-F_{N}\right\| \ll N^{-\bar{c}(\beta)}(\log N)^{\frac{\log (1 / \beta)}{\log 2}},
$$

where

$$
\bar{c}(\beta)=\frac{\log (1 / \beta) \log (2)}{\log (4 / \beta) \log (2 / \beta)+\log (2)^{2}} .
$$

Proof (Tenenbaum). We first observe that $Q_{F}(1 / T)$ can be upper bounded by the concentration function $Q_{G_{M}}(1 / T)$ associated to the convolution of independent Bernoulli random variables $X_{n}$, $0 \leq n \leq M$, with $\mathbb{P}\left[X_{n}=0\right]=\mathbb{P}\left[X_{n}=\beta^{n}\right]=\frac{1}{2}$. We choose $M=\lfloor\log (T) / \log (1 / \beta)\rfloor$ so that $\beta^{M} \geqslant 1 / T$. Next let $R=1+\lfloor\log (4) / \log (1 / \beta)\rfloor$, so that $\beta^{R}<1 / 4$. Then $Q_{G_{M}}(1 / T)$ is also the concentration function associated to the convolution of the laws $F_{a}, 0 \leq a<R$, where $F_{a}$ is the law of

$$
\sum_{0 \leq n \leq M, n \equiv a \bmod R} X_{n}
$$

However, for each $a$, the values of these random variables are ordered lexicographically and the gaps are $>\beta^{M} \geqslant 1 / T$. So we have $Q_{F_{a}}(1 / T) \ll 2^{-M / R}$. Since the concentration of a convolution product does not exceed that of the factors, we get

$$
Q_{F}(1 / T) \leq Q_{F_{a}}(1 / T) \ll 2^{-\log (T) /(\log (1 / \beta)(1+\log (4) / \log (1 / \beta)))}=T^{-c_{0}(\beta)},
$$

where

$$
c_{0}(\beta)=\frac{\log 2}{\log (4 / \beta)} .
$$

According to (2.10) we, thus, have (with $L=\left\lfloor\log _{2} N\right\rfloor$ and $h=\left\lfloor\log _{2}(T \log T)\right\rfloor$ )

$$
\left\|F-F_{N}\right\| \ll T^{-c_{0}(\beta)}+T \beta^{L-h} \ll T^{-c_{0}(\beta)}+T^{1+\frac{\log (1 / \beta)}{\log 2}} N^{-\frac{\log (1 / \beta)}{\log 2}}(\log T)^{\frac{\log (1 / \beta)}{\log 2}} .
$$

Hence by choosing

$$
T=N^{\frac{\log (1 / \beta) / \log 2}{1+\log (1 / \beta) / \log 2+c_{0}(\beta)}}
$$

we finally obtain

$$
\left\|F-F_{N}\right\| \ll N^{-\frac{c_{0}(\beta) \log (1 / \beta) / \log 2}{1+\log (1 / \beta) / \log 2+c_{0}(\beta)}}(\log N)^{\frac{\log (1 / \beta)}{\log 2}}=N^{-\bar{c}(\beta)}(\log N)^{\frac{\log (1 / \beta)}{\log 2}}
$$

as proposed. 